University of Zurich

Department of Economics

Working Paper Series

ISSN 1664-7041 (print)

ISSN 1664-705X (online)

Working Paper No. 402

\title{
Optimal Short-Time Work: Screening for Jobs at Risk
}

Julian Teichgräber, Simon Žužek and Jannik Hensel

February 2022 


\section{Optimal Short-Time Work: Screening for Jobs at Risk ${ }^{*}$}

\author{
Julian Teichgräber \\ University of Zurich
}

\author{
Simon Žužek \\ University of Zurich
}

February 7, 2022

\author{
Jannik Hensel \\ University of Zurich
}




\section{Introduction}

Lockdowns and temporary closures in the wake of the recent Coronavirus pandemic have led many governments to increase the scope and funding of job retention programs to prevent a sudden and massive rise in unemployment. A common characteristic of these programs is the introduction of short-time work (STW), under which governments subsidize temporary hour reductions of firms. During recessions, STW programs can become substantial in size. As an example, 6 million individuals or $13 \%$ of the workforce have received subsidies from the German "Kurzarbeit" in April 2020 after the scope of the existing program has been expanded quickly. In a similar fashion, the program has been used by 1.1 million German workers in the aftermath of the financial crisis in 2009.

As with general job retention policies, the explicit purpose of STW programs is to reduce unemployment by means of sustaining employment in existing jobs. In practice, however, an external policy maker will find it difficult to determine which jobs are at risk of termination and should therefore receive subsidies. Similarly, not all jobs are viable in the long-run and some existing jobs with low economic prospects should rather be separated. The asymmetry of information between firms and the policy maker gives rise to adverse selection: for a given STW policy, some firms will benefit from subsidies for hour reductions without any benefit to the public in terms of saved jobs.

In this paper, we use a mechanism design approach to analyze the optimal job retention policy in the face of asymmetric information about match productivity. Importantly, we do not restrict the optimal solution to be an STW scheme a priori; instead, we allow the policy maker to choose any assignment of hours and subsidies as long as private incentives are respected. We then show that hour reductions arise as an optimal solution precisely because of the asymmetric information problem. The intuition for our result is that more productive firms also stand more to lose when asked to reduce hours. Thus, hour reductions of STW policies can be used to screen for jobs at risk and mitigate adverse selection.

Our explanation of STW as a screening tool is new compared to the existing literature, which mostly emphasizes a combination of wage-bargaining and liquidity frictions to motivate why the government should subsidize hour adjustments between firms and workers. Parts of the welfare-improving effects of STW in these models therefore always stem from correcting inefficient hours. In our model, on the other hand, firms and workers set efficient hours as long as they agree to continue the match. The market inefficiency which the planner tries to correct only arises on the extensive margin. Too many matches separate since the private and social costs of unemployment do not align, and a targeted subsidy to reduce the separation rate is infeasible due to asymmetric information. Instead, the social planner demands an hour-reduction to screen matches for their type. From the perspective of the planner, STW only serves as a screening mechanism that trades off production efficiency with the value of retrieving private information about matches.

Importantly, asymmetric information captures the problem that the planner cannot perfectly target the recipients of the subsidy and instead has to accept a degree of welfare loss due to subsidizing the wrong firms because of selection issues and private information. This is a commonly cited argument against employment protection in general and STW in particular ${ }^{1}$ and our approach allows us to formalize this trade-off.

Our model helps to understand the findings of recent empirical studies, which have mostly focused on direct employment effects to evaluate short-time work policies. An insight from our model is that focusing only on employment across firms can be misleading about welfare and optimality. As an example, consider the findings by Cahuc et al. (2021), who have documented that STW benefits are partly paid to firms with lower revenue shocks and no discernible effects on employment. Yet given the asymmetry of

\footnotetext{
${ }^{1}$ See for example Cahuc et al. (2021) for an empirical discussion.
} 
information about the shock and the screening role of hour reductions, it is optimal to include jobs that are not at risk of termination in the STW scheme. A lack of employment effects for some firms therefore cannot be interpreted as evidence for an inefficient policy. In fact, in our calibrated optimal policy more than $80 \%$ of matches in STW are adversely selected and exhibit no employment effects.

Our paper also relates to whether short-time work can serve as a natural extension to an existing unemployment insurance (UI) scheme. While UI aims at the extensive margin of job creation and destruction, STW can be viewed as an extension through which the policy maker can adjust the intensive margin of hours. In our model, offering STW allows the social planner to complement an existing UI scheme by reducing overall fiscal costs while keeping the utility value of UI constant for all workers.

To quantify the extent to which an optimal STW scheme complements an existing UI system, we calibrate our model to moments of the German labor market prior to the Great Recession. Before 2009, the scope of the German "Kurzarbeitsprogramm" was minuscule and a negligible factor in the overall labor market policy. Our quantitative exercise shows that an optimal STW policy would have strongly intervened in the labor market and would have reduced the separation rate by $16-33 \%$, or 1.2-2.4 percentage points.

Related Literature. A striking feature of the recent literature on STW is that there is no agreement on the underlying market inefficiency that STW addresses. Different proposals include human capital externalities (Tilly and Niedermayer, 2016) and liquidity constraints (Giupponi and Landais, 2018), all of which require wage bargaining frictions that prevent matches from reaching the socially efficient outcome privately. A main rationale for STW in their models is to alleviate these frictions. In contrast, we introduce a completely different explanation of short-time work as a screening tool based on the fact that the planner cannot distinguish job matches who need support from those who do not. The most important difference to these models is that we do not impose any frictions on the private decisions about hours or wages. Without any policy intervention, hours of firms and workers which do not separate are socially efficient, which is not the case in models with bargaining frictions.

Tilly and Niedermayer (2016) and Giupponi and Landais (2018) derive optimality conditions for replacement rates in linear STW policies. We take a more general approach by not restricting our policy design to linear policies and allowing for arbitrary subsidy schedules as a function of hours instead. In the estimation of Tilly and Niedermayer (2016), the optimal replacement rate is close to the boundary of one, indicating that a larger optimization space as in our approach may be important. Our non-linear solution space also reveals an important distinction vis-à-vis a linear policy. In order to facilitate reallocation and prevent adverse selection of low types into the STW program, our optimal policy induces a minimum threshold of hours worked. In our calibrated economy, for example, matches with a reduction of more than $28-45 \%$ relative to full-time work are not subsidized and separate instead and the average replacement rate relative to the unemployment benefit is $25.3-47 \%$.

The setup of our model is closely related to Cooper et al. (2017) and most recently Cahuc et al. (2021), who model a frictional labor market in which workers and firms jointly maximize the surplus derived from a match without subject to bargaining constraints. Similar to our approach, worker-firm matches which do not separate agree on socially efficient hours in the absence of any policy intervention. Differently to our approach, these papers do not discuss which STW policy would be optimal, but rather introduce a policy exogenously to study its effects quantitatively. In a similar fashion, Balleer et al. (2015) study the effect of altering eligibility criteria for STW.

Many of the existing papers try to estimate the degree of adverse selection into STW programs; either by low-productivity matches with negative effects on reallocation (Giupponi and Landais, 2018; Cooper et al., 2017) or high productivity matches with excessive costs and no employment effects (Cahuc et al., 
2021; Boeri and Bruecker, 2011). Those papers have in common that they observe negative selection effects from STW and compare it to the positive effect of saving jobs ${ }^{2}$. All of these papers highlight the information asymmetry between firms and policy makers regarding productivity as important concerns for efficiency of these programs. Yet as of today, no theoretical models have been proposed to study whether the incentive problems outweigh potential benefits of the program. Our paper is the first to account for adverse selection formally through a model of incomplete information. Because of that, we are able to shed light on these recent empirical findings. Importantly, we show that a focus on employment effects can be misleading since participation of high-productivity types happens even in an optimal policy and that a well designed STW policy is precisely the best way to mitigate adverse selection and sustain jobs at the lowest costs.

In our model, hour reductions act as a mechanism to screen for matches which are not at risk of termination. We therefore draw heavily from the theoretical screening literature initiated by Mussa and Rosen (1978). In our context, the private information of matches matters for the outside option - whether they would separate without any planner intervention. Our paper is therefore different to much of the standard screening models where private information matters for the intensive margin (e.g. "taste" or "ability") and the outside option is held constant. The most closely related theoretical paper is Jullien (2000), who was the first to study asymmetric information with varying outside options. We follow his approach to state our model as an optimal control problem with state constraints. For a recent extension to models with nontrivial participation constraints and discontinuous objective functions, see Martimort and Stole (2020).

Our paper can be seen as an application of the screening literature to optimal labor market and job retention policies. In particular, this paper relates to Blanchard and Tirole (2008), who study the joint design of unemployment insurance and layoff taxes under different frictions, including unobserved match heterogeneity. In contrast, our model lets the optimal policy depend on the hours worked and imposes participation constraints for all types, therefore precluding any cross-subsidization from highto low-productivity matches. The use of work hours as a screening tool for productivity is recognized by Stantcheva (2014). She studies optimal redistributive income taxation in the presence of adverse selection where firms screen workers' private information. In our model, the social planner uses hours worked as a tool to screen firm-worker matches' underlying productivity.

The rest of the paper is structured as follows. In section 2, we introduce a labor market model with asymmetric information and socially excessive separations. We derive the solution under perfect information as a comparison. Section 3 discusses the optimal STW policy in our model and contains our main analytical results. Section 4 contains our calibration. Section 5 concludes.

\section{Model}

In this section, we present a model of a general job retention program with asymmetric information about match productivity. A policy maker would like to save jobs at risk, but firms and workers have private information about the viability of their match. In our model, the policy maker is free to offer subsidies for each individual match in the economy to maximize welfare. In addition, the planner may condition these subsidies on changes in working hours. Our model, therefore, spans a broad range of labor market policies, ranging from unconditional employment subsidies to STW schemes with non-

\footnotetext{
${ }^{2}$ Two papers estimate the causal effects of STW policies on employment and calculate the fiscal externality through behavioral responses. In Giupponi and Landais (2018) in the context of Italy, the estimate of the fiscal externality is larger than one, implying that more than one dollar in revenue needs to be raised to pay for one dollar in short time work. They attribute this to moral hazard effects of the policy. The results of Kopp and Siegenthaler (2021) for the case of Switzerland, on the other hand, point to a fiscal externality of less than one, meaning the policy even pays for itself.
} 
linear replacement rates, as they are often observed in European countries. In particular, many European countries limit STW by imposing both minimum and maximum reductions to working hours. ${ }^{3}$

Unlike simple unconditional wage subsidies, STW has the potential to screen for productivity using working hours. By requiring an hour reduction from firms participating in the program, the policy maker aims to dissuade high-productivity matches from participating because those matches are not truly at risk of separation. As an example, consider two worker-firm matches L (low) and $\mathrm{H}$ (high). Without an intervention, $\mathrm{L}$ would separate but $\mathrm{H}$ would not. If the policy maker could distinguish between both matches, she would offer $\mathrm{L}$ an employment subsidy to keep the match intact and would exclude $\mathrm{H}$ from participating. However, if firm information is private and hours cannot be changed, the policy maker would have to offer the same subsidy to both matches. With an STW policy, the policy maker could use the hour reduction to design a policy able to distinguish between the matches and target only $\mathrm{L}^{4}$. Since the shape of the policy in our model is unrestricted in transfers and hours, it is not immediate that the optimal policy resembles an STW program as they currently exist. For example, the optimal policy could be an employment protection subsidy without changes on the intensive margin as in Blanchard and Tirole (2008). As an extension in Appendix C, we show how an employment protection subsidy may arise optimally as a special case of our framework.

\subsection{Setup}

Existing matches. Matches are jointly formed by workers and firms and a firm consists of one worker. It is common knowledge to all agents that the match is subject to a productivity shock in the current period, where the productivity parameter $\theta$ is drawn from a distribution that follows the CDF $F$ with support on $[\underline{\theta}, \bar{\theta}]$ with $\underline{\theta} \geq 0$. As a technical assumption, we assume that $F$ has full support and an increasing hazard rate, $F$ is logconcave and twice continuously differentiable. ${ }^{5}$ It could be a severe disruption of the economy with a large variance as in the case of the Covid-19 pandemic or the Great Recession, or it could be a normal shock with less drastic effects. The production of a match further depends on hours $n$, which the firm and worker have to agree upon.

Once productivity is drawn, the value of the match from the perspective of the firm is

$$
J(\theta, n)=\theta \times n-w(\theta, n)
$$

where production is linear in hours $n$ and a match-specific wage $w$ is paid to the worker. Additionally, each firm may post vacancies to fill future jobs. Since there is free entry, the value of a vacancy $V$ is generally 0 . This represents the outside option of the firm.

For the worker, the corresponding value of the match is

$$
W(\theta, n)=w(\theta, n)-c(n)
$$

where $c(n)$ is the disutility that the worker bears for providing $n$ hours of labor to the match. We assume that $c(0)=0, c^{\prime}, c^{\prime \prime}>0$.

\footnotetext{
${ }^{3}$ For example, around the Great Recession in 2008-2009, some countries required hours to be reduced by at least $10 \%$ (as in Germany and Austria), or that hours be reduced by at most 50\% (as in the Netherlands and Luxembourg). See figure B.1 in the Appendix for an overview of STW hour ranges across OECD countries.

${ }^{4}$ Whether designing a policy that results in a subsidy to just $\mathrm{L}$ is indeed optimal depends on the distribution of productivity. This is a question that we tackle in our general setting.

${ }^{5}$ Logconcavity will allow us to abstract from bunching issues. This assumption first appeared in Lewis and Sappington (1989) in a slightly different formulation and later in Jullien (2000). Continuous differentiability can be relaxed, for instance to upper semicontinuity of $F$, but makes the characterization of Theorem 1 technically more difficult.
} 
Reallocation. When unemployed, the worker receives unemployment benefits $b$ and searches for new employment options with an expected value of $R$. In our model, $R$ embodies the idea that job search leads to new options, which create value to the economy by reallocating resources. We therefore also refer to $R$ as the reallocation value. Unemployment benefits and the reallocation value jointly promise the worker a value of unemployment $U$ of

$$
U=b+R
$$

For our purposes, we assume that the unemployment program is pre-existing and cannot be changed and therefore benefits $b>0$ are fixed. The parameter $R$ is fixed, too, since we do not model the reallocation value explicitly. Hence the value of unemployment $U$ can be treated as a promise that the social planner implicitly made to the worker. ${ }^{6}$

Laissez-faire outcome. To determine the wage, firms and workers enter into a bargaining game about the surplus of the match, defined as

$$
J(\theta, n)-V+W(\theta, n)-U
$$

The specific details of the bargaining game between firm and worker are irrelevant for the allocation in our model. Importantly, we assume that the value that each of the two receives is increasing in the total surplus, which would for example be satisfied under the commonly used Nash-bargaining rule. Therefore, we can assume that firm and worker jointly maximize the match value prior to bargaining. The surplus captures the benefits that both parties jointly derive from staying in the match in the current period. It is increasing in current productivity $\theta$ but decreasing in the value of unemployment $U$. The agents can agree to exercise their outside options - unemployment and posting a new vacancy - and dissolve the match. This decision is reflected by the choice of the inclusion variable $\iota$. Evidently, this happens in case the surplus becomes negative. We refer to the relevant surplus of the match in the absence of an intervention by the policy maker as the (match) outside option $S_{O}$ and write

$$
\begin{aligned}
S_{\mathrm{O}}(\theta) & =\max _{n \geq 0, \iota \in[0,1]} \iota *(J(\theta, n)-V+W(\theta, n)-U) \\
& =\max _{n \geq 0, \iota \in[0,1]} \iota *(\theta n-c(n)-U)
\end{aligned}
$$

The outside option surplus is $S_{\mathrm{O}}(\theta)$ increasing in $\theta$ with $S_{\mathrm{O}}(\underline{\theta})=0$. The maximization problem for the outside option allows for stochastic match dissolution (i.e. $\iota \in[0,1]$ ) but it always takes deterministic boundary values of either 1 or 0 , depending on whether the surplus from maintaining the match is positive or not. More precisely, hours $\hat{n}(\theta)$ which matches of type $\theta$ set in the outside option are

$$
\hat{n}(\theta)= \begin{cases}0 & \text { if } \theta<\theta_{0} \\ \left(c^{\prime}\right)^{-1}(\theta) & \text { if } \theta \geq \theta_{0}\end{cases}
$$

and $\hat{\imath}(\theta)=\mathbb{1}_{\left\{\theta \geq \theta_{0}\right\}}(\theta)$ where $\theta_{0}$ satisfies $\theta_{0}\left(c^{\prime}\right)^{-1}\left(\theta_{0}\right)-c\left(\left(c^{\prime}\right)^{-1}\left(\theta_{0}\right)\right)=U$. $^{7}$ Type $\theta_{0}$ is a threshold productivity that satisfies $S\left(\theta_{0}\right)=0$. Matches with a productivity parameter below $\theta_{0}$ will exit whereas matches with higher productivity will stay intact. Notably, the match outside option $S_{O}$ is always weakly positive

\footnotetext{
${ }^{6}$ We assume that the reason why unemployment insurance exists is exogenous to our model. To determine the joint design of unemployment benefits and STW, one would have to be specific about the role of unemployment benefits in this economy, for example as an insurance to idiosyncratic risk. Incorporating this into our model would be an interesting future extension.

${ }^{7}$ The exit cutoff $\theta_{0}$ is well defined because $c$ is strictly convex.
} 
whereas the match surplus may turn negative.

To make our problem nontrivial, we assume that there would always be matches that exit absent a social planner intervention. Formally, we assume that $\underline{\theta}\left(c^{\prime}\right)^{-1}(\underline{\theta})-c\left(\left(c^{\prime}\right)^{-1}(\underline{\theta})\right)<U$.

Social planner objective. The decision to dissolve a match, however, is not necessarily socially efficient even if it avoids a negative value of $S_{\mathrm{O}}$. The reason is that part of the outside option of the worker is funded through an existing unemployment insurance scheme. We assume that unemployment benefits have to be financed through general public funds at marginal costs larger than unity. This captures the general fact that revenue of the government, derived through taxes, tariffs or other means, always imposes additional social costs in collection. We impose therefore that any transfer $t$ that the planner makes to the match - including the exogenous unemployment insurance - enters the welfare function with costs $(1+\tau) \times t$, where $1+\tau$ are the marginal costs of public funds (Laffont and Tirole, 1990). The marginal cost of these public funds is larger than one, capturing the fact that raising government revenues always introduces some distortion. Unemployment insurance, therefore, induces additional social costs and the policy maker has an incentive to lower the bill of unemployment.

The social planner can introduce a policy to address this issue of inefficient and costly Laissez-faire separation. A policy in our model takes the form of a set of alternative contracts which the policy maker offers to firms and workers.

Definition 1. A policy is a set of contracts $\{n(\theta), \iota(\theta), t(\theta)\}_{\theta \in[\underline{\theta}, \bar{\theta}]}$ with $n:[\underline{\theta}, \bar{\theta}] \rightarrow \mathbb{R}^{+}, \iota:[\underline{\theta}, \bar{\theta}] \rightarrow[0,1]$ and $t:[\underline{\theta}, \bar{\theta}] \rightarrow \mathbb{R}$.

Intuitively, a policy consists of a schedule of social planner transfers which are conditional on hours worked and the decision to separate or not. ${ }^{8}$ For a productivity type $\theta$, a policy specifies i) hours $n(\theta) \in$ $\mathbb{R}^{+}$, ii) inclusion $\iota(\theta) \in[0,1]$, which determines whether a match is sustained or separated and iii) transfers $t(\theta) \in \mathbb{R}$. A policy then induces a match specific policy surplus

$$
S_{\mathrm{P}}(\theta)=\iota(\theta) \theta n(\theta)+t(\theta)-c(n(\theta))+(1-\iota(\theta)) R-U
$$

The exact bargaining protocol for how to share the surplus that includes social planner transfers is irrelevant as long as worker and firm surplus are both monotone increasing in the match surplus. In particular, whether transfers are directed to the worker or to the firm plays no role because the bargaining dynamics completely absorb any such distinction.

The social planner objective consists of two components. On the one hand, the planner wants to maximize the surplus of matches $S_{\mathrm{P}}$ through its alternative offer. On the other hand, matches do not internalize the costs of unemployment imposed by marginal costs of public funds and therefore the planner would like to reduce transfers to matches. Total welfare is then given by

$$
\mathcal{W}=\int_{\underline{\theta}}^{\bar{\theta}} \underbrace{S_{\mathrm{P}}(\theta)}_{\text {Surplus }}-\underbrace{(1+\tau) t(\theta)}_{\text {Cost of public funds }} d F(\theta) .
$$

Participation constraint. We also impose that firms and workers participate voluntarily in the offered policy. This assumption on individual rationality is an important distinction between employment subsidies such as STW and other settings. Matches in our policy cannot be made worse off than if they refused

\footnotetext{
${ }^{8}$ If productivity types $\theta$ are public knowledge, the definition of a policy which conditions on productivity types is without loss to maintain this intuitive interpretation as is the case under incomplete information by the revelation principle.
} 
to participate and continued on their own. Since the match outside option is increasing in productivity $\theta$, asking for voluntary participation of firms also precludes redistribution, which distinguishes our theoretical setting from e.g. the literature on optimal non-linear taxation. A further implication of individual rationality is that the subsidy cannot simply work as an insurance for economic shocks, since matches may always walk away ex-post. Formally, we write the individual rationality constraint as

$$
S_{\mathrm{P}}(\theta) \geq S_{\mathrm{O}}(\theta) \quad \forall \theta \in[\underline{\theta}, \bar{\theta}] .
$$

An important aspect of the individual rationality constraint is that it imposes that the planner has to keep her promise of delivering the outside option of unemployment. As an example, consider a single match with productivity 0 and reallocation value $R$. The surplus of the match is $S(0)=-U$, i.e. if the match continued it would forgo the flow value of unemployment this period. But the outside option of the match is $S_{O}(0)=0$. The assumption of individual rationality requires that the planner has to make the match indifferent.

To do so, the planner has - in essence - two options. She can either decide to save the match, in which case she offers a contract with $\iota=1$ and $t=U$, compensating the value of unemployment through transfers. The alternative option is to separate the match and release the worker into unemployment by setting $\iota=0$ and $t=b$. Separating the match reduces the required transfer to $b$, since the worker additionally receives the option value of a new job $R$.

\subsection{Efficient hours and transfers under complete information}

The inefficiency of private match decisions in the Laissez-faire setting comes from the fact that workers and firms do not internalize the social costs of the unemployment insurance scheme and the motive for government intervention is, therefore, to reduce these excess costs by reducing unemployment while maintaining the promised unemployment utility of $U$ for workers. To see how this objective relates to STW, it is helpful to consider the simple case where productivity $\theta$ is observed and the planner can assign working hours $n$ and transfers $t$ based on observed productivity directly. In addition, the social planner can also make a recommendation of whether to maintain or dissolve the match captured by $\iota$ in Definition 1 as is done by the match in the maximization problem for the outside option. Denote the value of $\theta$ that solves $\theta\left(c^{\prime}\right)^{-1}(\theta)-c\left(\left(c^{\prime}\right)^{-1}(\theta)\right)=R$ by $\theta_{\text {eff. }}$ Then, the social planner maximizes (W) by assigning working hours

$$
n_{\text {eff }}(\theta)= \begin{cases}\left(c^{\prime}\right)^{-1}(\theta) & \text { if } \theta \geq \theta_{\text {eff }} \\ 0 & \text { else }\end{cases}
$$

setting $\iota_{\mathrm{eff}}(\theta)=\mathbb{1}_{\left\{\theta \geq \theta_{\mathrm{eff}}\right\}}(\theta)$ and making transfers

$$
t_{\text {eff }}(\theta)= \begin{cases}b-\left(\theta n_{\text {eff }}(\theta)-c\left(n_{\mathrm{eff}}(\theta)\right)\right) & \text { if } \theta \leq \theta_{\text {eff }} \\ 0 & \text { else }\end{cases}
$$

in order to fulfill the (IR) constraint.

We call $n_{\text {eff }}$ the production-efficient working hours because they maximize output minus disutility of labor. The transfers under complete information $t_{\text {eff }}$ are such that (i) matches receive unemployment benefits when production is inefficient (ii) matches receive the difference between unemployment benefits and the value of efficient production to the match when the value of efficient production is positive but below unemployment, and (iii) transfers are zero when matches prefer to work at the efficient level instead of dissolving the match for unemployment benefits. Importantly, if $R>0$, it holds that $\theta_{\text {eff }}<\theta_{0}$. 
Since the value of reallocation $R$ is not internalized by matches, there will be inefficient separation by matches absent any policy intervention. The social optimum asks all matches to work at their efficient level of hours and incentivizes them to do so by making everyone at least as good off as in unemployment.

Figure 1 provides a graphical comparison of the optimal social planner intervention under complete information to working hours and transfers of matches in the outside option. Panel 1a shows that working hours coincide on $\left[\underline{\theta}, \theta_{\text {eff }}\right]$, when separation is efficient, and on $\left[\theta_{0}, \bar{\theta}\right]$, when matches work at an efficient level without intervention. Transfers to guarantee at least the value of unemployment can be seen in panel 1b. Hence, policy $\left\{n_{\text {eff }}, \iota_{\text {eff }}, t_{\text {eff }}\right\}_{[\theta, \bar{\theta}]}$ is not an STW policy and rather an unemployment policy because it only shifts the extensive margin of unemployment, but does not change the intensive margin of hours when matches are maintained.

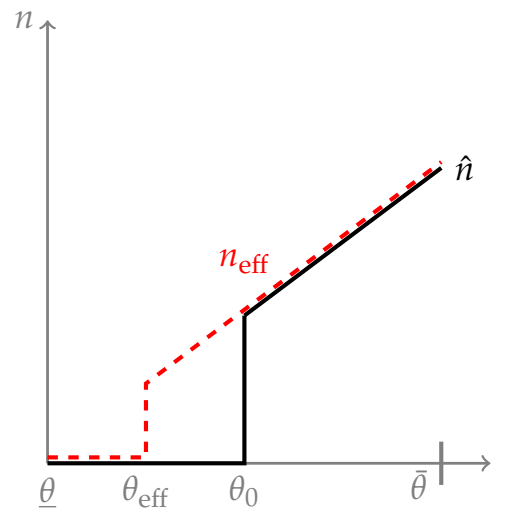

(a) hours

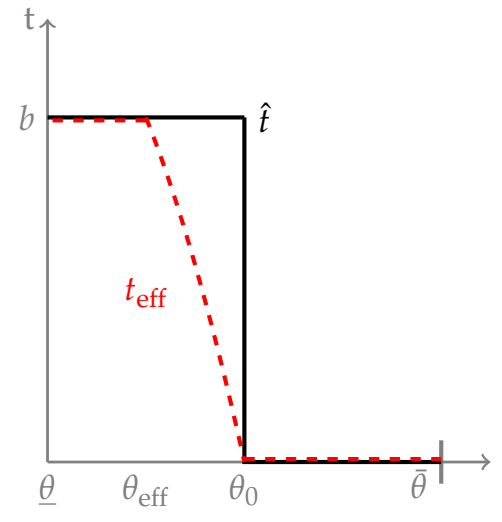

(b) transfers

Figure 1: Hours and transfers for matches in the outside option (solid) and the perfect-information allocation (dashed). The example uses a quadratic function for the disutility of labor.

\subsection{Introducing incomplete information}

When match productivity is unobserved to the social planner, she cannot directly mandate the ideal flexible unemployment policy from the previous section. Instead, the social planner needs to elicit the productivity parameter of a match and can offer a policy based on the reported type.

In the presence of private information, the policy maker must offer all matches in the economy the same conditions irrespective of their idiosyncratic productivity draw. Firms and workers then have the option to select into a specific contract with accompanying hours and transfer that maximize their surplus. From the perspective of the match, a contract $\{n(\tilde{\theta}), t(\tilde{\theta}), \iota(\tilde{\theta})\}$ implies a policy surplus depending on the reported productivity type $\tilde{\theta}$ and true type $\theta$.

$$
S_{\mathrm{P}}(\tilde{\theta} \mid \theta)=\iota(\theta) \theta n(\tilde{\theta})+t(\tilde{\theta})-c(n(\tilde{\theta}))+(1-\iota(\tilde{\theta})) R-U
$$

(Policy surplus)

Note that with private information, the policy maker has to take into account the incentives of all matches to misreport their productivity as $\tilde{\theta} \neq \theta$ is generally possible. If the planner offers a high subsidy to matches with low productivity, she has to take into consideration that matches with higher productivity can misreport and claim the same transfers. Here, conditioning on a reduction of working hours will help to differentiate since high productivity matches have more surplus to lose from an hour reduction than low productivity matches. As the policy maker can strategically use the contract offers to elicit private information, the tools available to the social planner are screening mechanisms. Part of the 
purpose to the policy maker of a job retention policy or STW, in particular, is therefore to improve the allocation of funds by screening for private information of firms. ${ }^{9}$

By the revelation principle, it is without loss to focus on direct mechanisms that ask for the underlying type $\theta$ instead of asking matches to select hours $n$ from the offered policies such that we can still define a policy as in Definition 1. All we need is to require that matches have no incentive to misreport their type. This incentive-compatibility constraint takes the form

$$
S_{\mathrm{P}}(\theta)=S_{\mathrm{P}}(\theta \mid \theta) \geq S_{\mathrm{P}}(\tilde{\theta} \mid \theta) \quad \forall \theta, \tilde{\theta} .
$$

We believe that the participation (IR) and incentive-compatibility constraint (IC) capture key features of labor market interventions such as employment subsidies and STW which are usually overlooked but help to understand what challenges these policies face. On the one hand, individual rationality relates to the fact that employment protection programs such as STW cannot be forced upon workers and firms. If a firm has no interest in participating and rather continues its business as usual, little can or should be done to change that. It further implies that such a program cannot act as an insurance, in which unaffected firms foot the bill for their troubled competitors. ${ }^{10}$ Incentive compatibility, or private information, on the other hand, relates to the fact that the recipients of subsidies can never be hand-picked individually, and instead, the policy maker must consider private incentives of misreporting in the design of an optimal policy.

To summarize, the social planner offers a policy in the form of a tuple of functions $\{n(\theta), \iota(\theta), t(\theta)\}_{\theta \in[\theta, \bar{\theta}]}$ to the match. Both hours $n$ and transfers $t$ are unrestricted in shape and could take a non-linear form. Layoffs can be stochastic and are governed by $\iota$. As a restriction, however, they are feasible feasible if only if they satisfy the incentive compatibility and participation constraints. Definition 2 formalizes the concept.

Definition 2. Policy $\{n(\theta), \iota(\theta), t(\theta)\}_{\theta \in[\theta, \bar{\theta}]}$ with $n:[\underline{\theta}, \bar{\theta}] \rightarrow \mathbb{R}^{+}, \iota:[\underline{\theta}, \bar{\theta}] \rightarrow[0,1]$ and $t:[\underline{\theta}, \bar{\theta}] \rightarrow \mathbb{R}$ is feasible if it satisfies (IC) and (IR).

The social planner looks to maximize the welfare objective via a feasible mechanism.

Definition 3. A feasible mechanism $\{n(\theta), \iota(\theta), t(\theta)\}_{\theta \in[\underline{\theta}, \bar{\theta}]}$ is optimal if it maximizes (W).

\subsection{Suboptimality of production efficient working hours}

The efficient production that would be implemented under complete information is a key benchmark in the model of incomplete information. The social planner wants to maximize the match surplus while transfers come at a social cost. In addition, holding transfers constant, the match surplus increases if the implemented working hours get closer to efficient production. A natural question to ask is whether the social planner simply wants to implement efficient production under incomplete information as well.

The answer is no. Our next result states that it can never be optimal to implement production efficient working hours if there are strictly positive unemployment benefits.

Proposition 1. For any optimal policy $\left\{n^{*}(\theta), \iota^{*}(\theta), t^{*}(\theta)\right\}_{\theta \in[\theta, \bar{\theta}]}$, it can never hold that $n^{*}(\theta)=n_{\text {eff }}(\theta)$ and $\iota^{*}(\theta)=\iota_{\text {eff }}(\theta)$ for all $\theta \in[\underline{\theta}, \bar{\theta}]$.

\footnotetext{
${ }^{9} \mathrm{~A}$ crucial assumption to make screening through hours possible is disutility from working of the employee. Otherwise the decision to work becomes binary, which we show in the extension in Appendix C.

${ }^{10}$ Unemployment insurance is a counter-example of a common labor market policy with involuntary participation in most developed countries.
} 
The intuition for this result is that the implementation of efficient production is too costly for the social planner. The cheapest policy that implements efficient production is by paying the same transfer $U$ to all matches. This just incentivizes all matches to operate at their efficient level of working hours. We provide a constructive proof of suboptimality by suggesting a modified policy that sets hours to zero for a sufficiently small share of match types for which it is just efficient to have positive hours. The social planner can then save on transfers for all higher types. The loss in production on types that would otherwise generate only just positive output is of second order. Hence, saving on incentive costs for high types dominates the loss in production for types that are just on the margin of efficiently operating with positive hours.

The suboptimality of implementing efficient production provides an insight on how a job retention scheme can be misdirected. The social planner should not aim to set transfers such that the efficient production is reached. Instead, the trade-off between the value of production through incentives via additional transfers and the social cost of spending must be taken into account carefully. The result also shows the key role of transfers. Their purpose is to incentivize matches to comply with the policy, but they are costly and should be implemented with care. Most importantly, the social planner should try to avoid making transfers to high types who would not need monetary incentives for efficient production without the policy.

\section{Optimal Policy}

We now turn to the analysis of the optimal policy.

\subsection{Characterization}

Information rent. Before we state the formal characterization of the optimal job retention policy, we want to provide some intuition for the result. First, we describe a necessary condition that relates optimal hours $n$ and policy inclusion $\iota$ to the incentive and participation constraints.

Proposition 2. Under incomplete information, hours $n(\theta)$ and inclusion $\iota(\theta)$ in the optimal feasible policy satisfy

$$
\left(n^{*}(\theta), \iota^{*}(\theta)\right) \in \arg \max _{(n, \iota) \in[0, \infty) \times[0,1]} \mathcal{V}=\left\{\iota n\left(\theta-\frac{\tau}{1+\tau} \frac{\Lambda^{*}(\theta)-F(\theta)}{f(\theta)}\right)-c(n)+(1-\iota) R-U\right\}
$$

where $\Lambda^{*}(\theta)$ is a Lagrange multiplier on the participation constraint (IR).

This result is derived analogously as Theorem 1 in Jullien (2000).

The optimality condition in Proposition 2 is instructive about incentives in designing the optimal job retention scheme. First, the policy inclusion variable $\iota$ takes a very simple structure. As $\mathcal{V}$ is linear in $\iota$, it will always take boundary values $\iota \in\{0,1\}$. There will be no stochastic layoffs and any optimal policy is deterministic. Matches are not simply maintained when their social value of inclusion is higher than the value of reallocation $R$. The decision to separate depends not only on output and direct payments to a given type, but also on incentive costs that a type incurs on other types. The optimal policy is also deterministic because transfers for given types are deterministic by assumption. In our model, transfers do not depend on the realization of the inclusion variable $\iota$, i.e. they are interim payments rather than ex-post payments. A model where transfers can be conditional on the realization of inclusion would not lead to different results because firms and workers are both risk-neutral expected utility maximizers. 
We can decompose the $\frac{\tau}{1+\tau}$-weighted marginal value of hours for type $\theta$

$$
\begin{aligned}
& \theta-\frac{\tau}{1+\tau} \frac{\Lambda^{*}(\theta)-F(\theta)}{f(\theta)} \\
= & \frac{1}{1+\tau} \theta-\frac{\tau}{1+\tau}\left(\theta-\frac{\Lambda^{*}(\theta)-F(\theta)}{f(\theta)}\right) .
\end{aligned}
$$

which consists of the direct contribution of an increase in hours for type $\theta$ to production minus the incentive costs imposed on all types of higher productivity. ${ }^{11}$ The relative weight of production on a type's social value is $\frac{1}{1+\tau}$ while the relative weight of transfers on the social value is $\frac{\tau}{1+\tau}$. An increase in expenditures $\Delta t$ only comes at marginal social cost $\tau$ because it raises the surplus by $\Delta t$ and incurs costs of public funds $(1+\tau) \Delta t$ such that the net effect is $-\tau \Delta t$.

The standard interpretation by Mussa and Rosen (1978) and its extension to a setting with nontrivial participation constraints by Jullien (2000) still applies and we extend it for the trade-off of production and costs of public funds in our model. A marginal increment $\Delta n$ in hours for a match of type $\theta$ increases output by $\theta f(\theta) \Delta n$ weighted at $1 /(1+\tau)$. In addition, The social planner can reduce transfers to type $\theta$ by $\theta \Delta n$ while (IC) is still satisfied for type $\theta$ which saves transfers of relative social value $(\tau /(1+\tau)) \theta \Delta n$. In short, an incremental increase in hours for type $\theta$ has relative social value $\theta f(\theta) \Delta n$ because it changes production and expenditures for type $\theta$ with the same magnitude.

The Lagrange multiplier $\Lambda^{*}$ also allows for an interpretation. In short, $\Lambda$ will denote the share of types that receive a transfer from the social planner, either through unemployment benefits or subsidies through the policy. For any match type $\theta$ where the constraint on the lower bound of hours $n \geq 0$ is not binding, $\Lambda^{*}(\theta)-F(\theta)$ denotes the mass of match types above $\theta$ for which the participation constraint (IR) binds. If the hours' constraint binds, i.e. $n=0$, the incentive costs of a marginal increase in production are not well-defined anymore and $\Lambda^{*}$ is not unique. This happens precisely for types at the bottom of the productivity distribution which will be separated to exploit the value of reallocation. With an understanding of $\Lambda^{*}$ at hand, the reformulation of $\operatorname{costs} \theta-\frac{\Lambda^{*}-F(\theta)}{f(\theta)}$ becomes clear. Any marginal change in hours $\Delta n$ incurs incentive costs as transfers need to be increased by $\Delta n$ for the mass $\Lambda^{*}(\theta)-F(\theta)$ of types above $\theta$ where the participation constraint (IR) is slack. Hence, expenditures from a marginal change in hours for type $\theta$ are the savings from increased output minus incentive costs on all higher types where the incentive constraint binds, i.e. $\left(\theta-\frac{\Lambda^{*}(\theta)-F(\theta)}{f(\theta)}\right) \Delta n$ at relative weight $\frac{\tau}{1+\tau}$.

If $\Lambda^{*}(\theta)=F(\theta)$, then a change in hours for type $\theta$ imposes no incentive costs above since the participation constraint must bind for all higher types. A marginal change in hours $\Delta n$ will only allow to reduce transfers on match type $\theta$ by $\Delta n$ as well (or even render transfers negative).

Moreover, on any interval where the incentive constraint binds rather than the participation constraint, we have $\Lambda^{*}$ since the mass of types above for which (IR) changes is the same on such an interval. We will see that there is exactly one such interval for which the planner optimally implements STW to interfere with the private decisions of firms and workers, i.e. hour decisions have to be changed through monetary incentivization such that (IC) binds rather than (IR). Match types below separate into unemployment while matches above are unaffected by the policy.

Characterization Result. The main result of this paper is a characterization of the optimal policy and its implications for the design of job retention schemes. The following further characterization marks the departure from Jullien (2000). Typically, only a subset of firm-worker matches select into existing STW

\footnotetext{
${ }^{11}$ As far as we know, the weighted virtual valuation was first introduced in ? where it appears through a Lagrange multiplier on the condition of budget balancedness.
} 
policies in practice. We show that an optimal policy induces a change in hours for a subset of intermediate types $\left[\theta_{1}, \theta_{2}\right] \subset[\underline{\theta}, \bar{\theta}]$ of types. This subset is an interval because only the highest and lowest types of the productivity distribution are not affected by the optimal policy. On the one hand, the match types with the highest productivity select hours at the efficient level and their incentives are maintained even with the additional contractual options added by the policy. Incentive costs never lead to any distortion of efficient production at the top and the highest productivity types would produce efficiently even in the absence of a policy. On the other hand, types of lowest productivity still separate. Some layoffs at the bottom of the productivity distribution are socially efficient and the social planner can maintain their incentives to opt for separation. Not only would production by them be socially inefficient but a subsidy would also only increase incentive costs for higher types.

However, there are still adverse selection issues for intermediately low and high types under the optimal policy which we discuss after formally stating the main characterization result.

The policy designer, therefore, faces a decision to find the exact intermediate interval $\left[\theta_{1}, \theta_{2}\right]$ where the policy induced hours $n^{*}$ differ from the outside option to screen for types where the change in hours has positive social value. As becomes evident from Theorem 1 , given a lowest type $\theta_{1}$ above which the policy assigns positive hours worked $n^{*}>0$, the incentive constraint tells us exactly the highest type $\theta_{2}$ below which the hours $n^{*}$ induced by the policy differs from the outside option $\hat{n}$. This is related to how a policy designer might intuitively approach the issue. How much unemployment is acceptable and what job retention policy achieves this target? The structure of incentives then naturally determines which share of the labor market selects into STW given the target on unemployment. Such an intuition also applies to the optimal policy, but its characterization is a bit more involved since the target separation rate is an endogenous parameter to our model. The choice of threshold values $\theta_{1}$ and $\theta_{2}$ is implicitly performed through the choice of a single Lagrange multiplier constant $\Lambda$. This Lagrange multiplier already appeared in Proposition 2 as a function $\Lambda^{*}(\theta)$ of $\theta$. This function is constant on $\left[\theta_{1}, \theta_{2}\right]$ and it turns out that it suffices to find the value of $\Lambda^{*}$ on this interval to fully characterize the optimal policy.

We are now in a position to formally state the main characterization.

Theorem 1. An optimal feasible policy $\left\{n^{*}(\theta), \iota^{*}(\theta), t^{*}(\theta)\right\}_{\theta \in[\theta, \bar{\theta}]}$ exists and it is unique. It satisfies

$$
\begin{gathered}
n^{*}(\theta)= \begin{cases}0 & \text { for } \theta \leq \theta_{1} \\
c^{\prime-1}\left(\theta-\frac{\tau}{1+\tau} \frac{\Lambda-F(\theta)}{f(\theta)}\right) & \text { for } \theta_{1}<\theta<\theta_{2} \\
c^{\prime-1}(\theta) & \text { for } \theta>\theta_{2}\end{cases} \\
\iota^{*}(\theta)= \begin{cases}0 & \text { if } \theta \leq \theta_{1} \\
1 & \text { if } \theta>\theta_{1}\end{cases}
\end{gathered}
$$

where $\theta_{1}$ is implicitly defined by

$$
\theta_{1}-\frac{\tau}{1+\tau} \frac{\Lambda-F\left(\theta_{1}\right)}{f\left(\theta_{1}\right)}=\theta_{e f f}
$$

while

$$
\theta_{2}=F^{-1}(\Lambda)
$$

and $\Lambda \in[0,1]$ is the unique solution to

$$
\int_{\theta_{1}}^{F^{-1}(\Lambda)} c^{\prime-1}\left(\theta-\frac{\tau}{1+\tau} \frac{\Lambda-F(\theta)}{f(\theta)}\right) d \theta=S_{O}\left(F^{-1}(\Lambda)\right)
$$




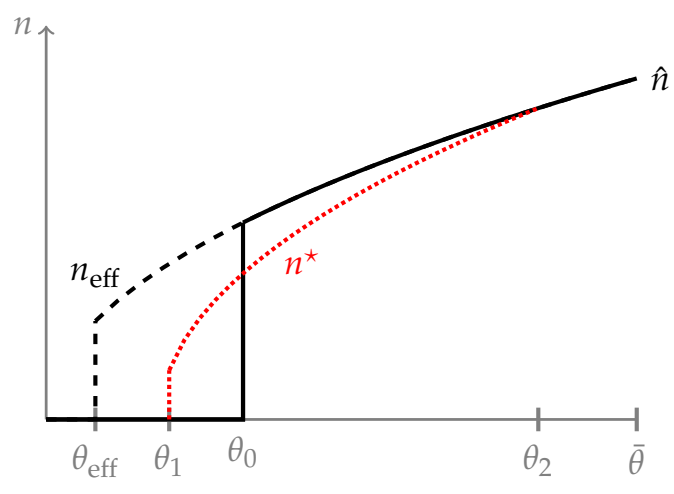

Figure 2: Optimal policy: hours $n^{*}$

Separation of low types. As should come at no surprise, matches below threshold $\theta_{1}$ are dissolved to make use of the social reallocation value $R$. They would simply not be productive enough such that the social planner rather pays unemployment benefits $b$ to them. An illustration of this separation cutoff can be found in Figure 3.

Cutoffs $\theta_{1}, \theta_{2}$. For match types above $\theta_{1}$, hours are such that the marginal disutility of labor equals the $\tau /(1+\tau)$-weighted marginal value of hours. Types above $\theta_{2}$ work efficient hours and receive no subsidy such that their $\tau /(1+\tau)$-weighted marginal value of hours is equal to their type, i.e. their marginal productivity in hours. In practice, STW policies do not affect the highest types of the productivity distribution and are more directed at lower parts of the productivity distribution where jobs are at risk. We observe a similar feature for the optimal policy in this model in the sense that jobs in the upper parts of the productivity distribution play no role in the policy design.

Incentive costs are a local property and a marginal change in hours incurs costs for all types above which are already affected by the policy and types who are on the margin of being affected.

Hence, a social designer who constructs a job retention scheme only needs to look at incentives locally of types whose behavior is changed by the policy and types on the margin of selecting into it.

In our setting, the share of agents at the top of the distribution which choose efficient hours according to their outside option is $1-\Lambda$. Thus, $\Lambda$ identifies the threshold $\theta_{2}$.

The other threshold $\theta_{1}$ below which matches are separated is identified through (8). Type $\theta_{\text {eff }}$ is defined

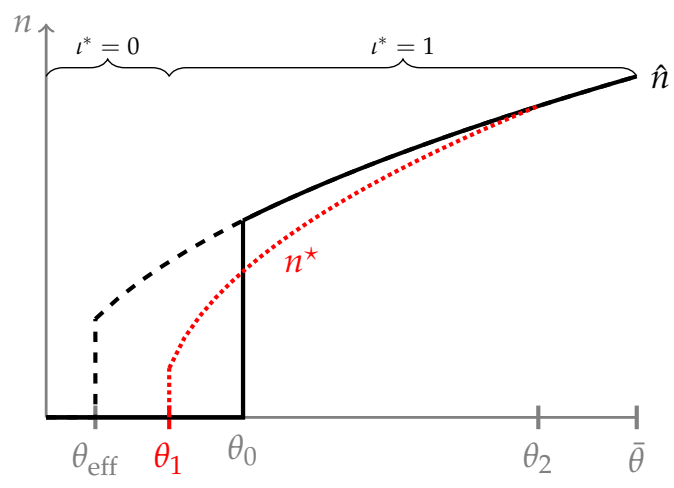

Figure 3: Illustration of optimal inclusion $\left(\iota^{*}=1\right)$ and separation $\left(\iota^{*}=0\right)$ 


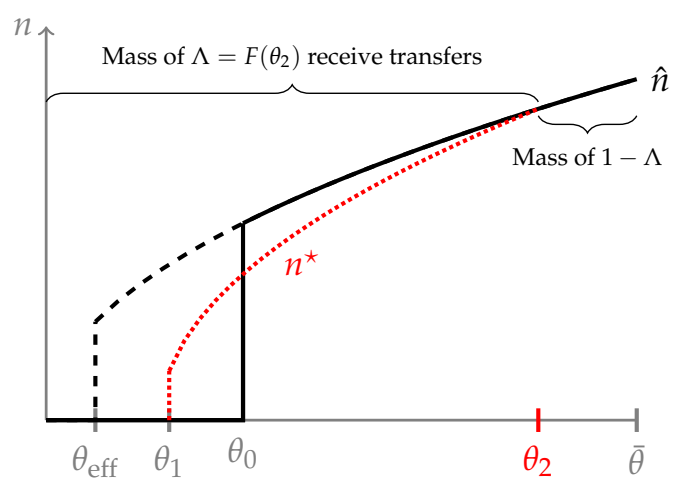

Figure 4: Optimal multiplier $\Lambda$

as the lowest type for which the social surplus with production weakly exceeds the value from reallocation $R$. Under asymmetric information, the $\tau /(1+\tau)$-weighted marginal value of type $\theta_{1}$ on the margin must just equal $\theta_{\text {eff }}$ such that its social value is weakly larger than separation and reallocation.

Lagrange multiplier $\Lambda$. The key question is how to find the parameter $\Lambda$ that determines cutoffs $\theta_{1}, \theta_{2}$ and the precise hours worked. For instance, an increase in $\Lambda$ leads to an upward shift of both $\theta_{1}$ and $\theta_{2}$. The identifying condition for $\Lambda$ in the optimal policy is (10). To understand this condition, it is instructive to understand the effects of $\Lambda$ on incentives. If $\Lambda$ were too low, then many jobs are saved (i.e. $\theta_{1}$ low), but this incentivization of maintaining matches comes at a high price. All types which continue working at the efficient level (i.e. types above $\theta_{2}$ ) would have to be paid a subsidy to maintain their incentives to work efficient hours. If $\Lambda$ were chosen too high, then too few jobs are saved and the policy loses its positive implications from job retention ( $\theta_{1}$ high). In addition, it would induce a large share of types above $\theta_{0}$ to reduce working hours and therefore incur a high loss in output for high types as compared to a world without the policy. Optimality condition (10) therefore states how to balance these tradeoffs of costly job retention versus too much underproduction. Figure 4 illustrates the role of $\Lambda$.

Short-time work. The following observation motivates why the optimal policy here exhibits key similarities with existing STW schemes.

Proposition 3 (Short-time work). For an optimal policy, it holds that $n^{*}(\theta) \leq n_{\text {eff }}(\theta)$ for all $\theta \in[\underline{\theta}, \bar{\theta}]$ with $n^{*}(\theta)<n_{\text {eff }}(\theta)$ whenever $n^{*}(\theta) \neq \hat{n}(\theta)$.

The optimal policy always keeps hours worked weakly below the first-best. The inequality is strict whenever the policy leads to a change in hours worked compared to the outside option. This motivates the following terminology. We say that a match of type $\theta$ is in STW if $n^{*}(\theta) \neq \hat{n}(\theta)$. Similarly, a match of type $\theta$ is not in STW if $n^{*}(\theta)=\hat{n}(\theta)$. This case encompasses the two possibilities that either the match is dissolved voluntarily or that the firm-worker work arrangement is unaffected by the policy. Interestingly, no match will ever produce above the efficient level. Setting hours above the efficient level is never optimal because it creates extreme incentive costs for higher types who actually prefer such high hours in addition to the loss through inefficient hours. Hence, hours worked can only be below the first-best because the reduction in hours also reduces incentive costs incurred by higher types.

Existence of Adverse Selection: The limits of short-time work. The following proposition follows (almost) directly from Theorem 1. 


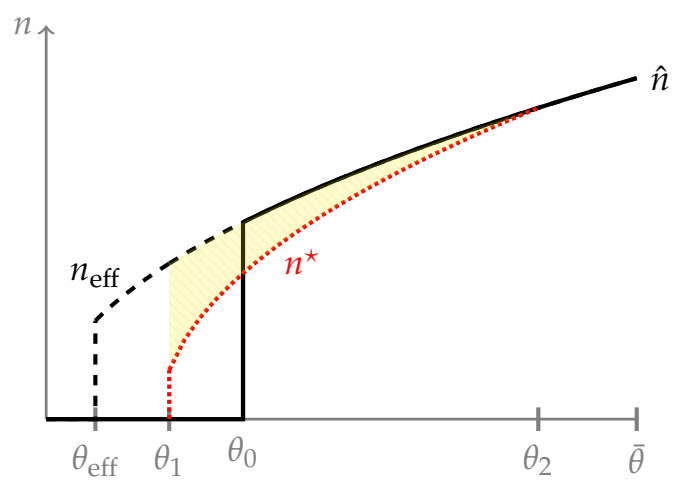

Figure 5: Underproduction through adverse selection

Proposition 4. It holds that $\theta_{2}>\theta_{0}$, i.e. there will always be a non-zero mass of matches selecting into STW although their outside option is to work efficient hours.

The innocuously looking property $\theta_{2}>\theta_{0}$ has a key implication for the limits of STW. Much of the recent literature has been concerned with the trade-off between saving jobs at risk and adverse selection by matches without need for government support. We show that there will always be adverse selection into STW in any optimal policy, i.e. there are always matches which are in STW $\left(\theta<\theta_{2}\right)$ although they would work efficient hours without the policy $\left(\theta>\theta_{0}\right)$. This adverse selection into underproduction is highlighted by the shaded area in Figure 5. The cost of eliminating adverse selection entirely is too high compared to the benefits it creates. As a consequence, the design of an optimal policy should always compare the welfare loss through adverse selection to the additional jobs saved rather than try to avoid adverse selection completely.

No distortion at the top (and at the bottom): The potential of short-time work. The well-known result of no distortion at the top from contract theory and the optimal tax literature extends to our model in a slightly modified way. In our setting, not only the highest productivity type produces at the socially efficient level, but all matches on the interval $\left[\theta_{2}, \bar{\theta}\right]$ do so. Hence, the optimal policy leads to no distortion for an interval at the top. Nevertheless, the length of the mass of types with no distortion depends on the policy itself.

In addition, we also obtain a no distortion at the bottom result from Proposition 3.

Proposition 5. It holds that $\theta_{1}>\theta_{\text {eff, }}$, i.e. matches who cannot achieve a value from output larger than reallocation value $R$ will be dissolved.

No match whose productivity shock is such that any positive production is socially inefficient will maintain the match. Matches are dissolved whenever it is socially efficient to rather opt for reallocation. Our model, therefore, states that a well designed policy can achieve to dissolve all inefficient matches, but not necessarily maintain all matches that are worth saving. Figure 6 gives an example of a suboptimal policy where matches are maintained that should rather be separated. In particular, this hints towards the fact that there is potential improvement for an STW policy whenever it suppresses reallocation below the efficient level. Well designed STW eliminates this type of adverse selection. However, optimal STW leads to too much unemployment rather than too little unemployment compared to the first-best.

Unemployment benefits $b$. The unemployment benefits $b$ do not appear in our characterization in Theorem 1 which might leave the reader wondering whether they are relevant to the optimal solution. It 


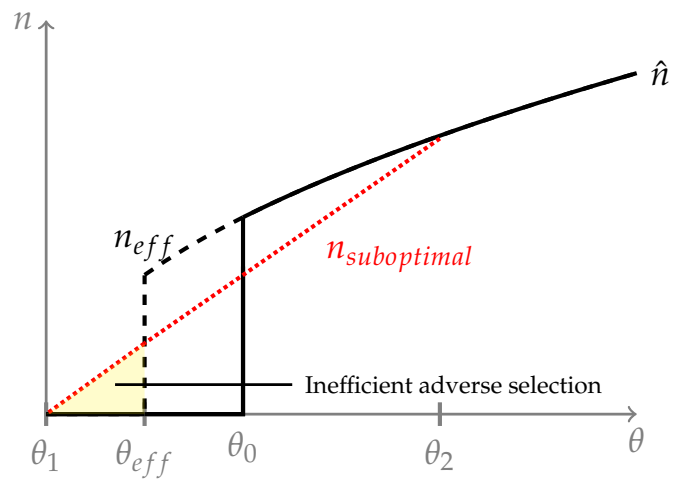

Figure 6: Example of suboptimal adverse selection in a short-time work policy

is therefore worth highlighting that the existence of unemployment benefits is the main inefficiency that makes STW optimal in our model.

Corollary 1. If there are no unemployment benefits, i.e. $b=0$, then the optimal policy is the laissez-faire policy $n^{*} \equiv \hat{n}, \iota^{*} \equiv \mathbb{1}_{\left\{\theta \geq \theta_{0}\right\}}, t^{*} \equiv 0$.

If $b=0$, the social planner's and the firm/worker incentives are perfectly aligned such that there is no need to intervene for the social planner. Firms and workers dissolve precisely the socially inefficient matches.

Transfers. So far, we have only discussed the structure of hours under the optimal policy. As is standard in such mechanism design models, the transfers can be immediately derived just from the incentive constraint given optimal hours $n^{*}$ and inclusion $\iota^{*}$.

Proposition 6. Transfers (or subsidies) under the optimal policy are

$$
t^{*}(\theta)= \begin{cases}b & \text { for } \theta \leq \theta_{1}, \\ c\left(n^{*}(\theta)\right)-\theta n^{*}(\theta)+\int_{\theta_{1}}^{\theta} n^{*}(x) d x+b & \text { for } \theta \in\left[\theta_{1}, \theta_{2}\right], \\ 0 & \text { for } \theta>\theta_{2} .\end{cases}
$$

The social planner transfers are of a simple form. Low productivity types on the interval $\left[\underline{\theta}, \theta_{1}\right]$ only receive the unemployment benefit and work zero hours. These are the matches that are not worth maintaining, but the social planner is still obliged to pay unemployment benefits due to the existing unemployment insurance program. The high productivity types $\left[\theta_{2}, \bar{\theta}\right]$ work at the efficient level as they would do without the policy. The policy is designed precisely in a way that no additional subsidies have to be paid for matches that do not select into STW. This is in contrast to a standard employment subsidy which cannot screen for jobs at risk and would need to subsidize even matches of highest productivity. ${ }^{12}$ In the Appendix, we discuss how a classical employment subsidy is nested in our model. The key deviation from the exposition presented here is that absent a convex disutility of labor, the planner can no longer use hours to screen for productivity.

A straightforward calculation shows that $t^{*}$ is a decreasing function in productivity $\theta$. Transfers to matches in STW are therefore always below the unemployment benefit and gradually decrease to zero for higher hours worked. At this point, it is worth highlighting that many STW policies employed in practice

\footnotetext{
${ }^{12}$ There have been efforts in countries like the Netherlands to target wage subsidies for those in need by conditioning the payment on the loss in sales (see OECD (2020)). These efforts can be seen as an attempt to receive an imperfect signal of match productivity.
} 
are a wage subsidy equal to the reduction in hours times unemployment benefits. ${ }^{13}$ In our optimal policy, unemployment benefits are indeed an optimal reference point for STW payments, although transfers are not necessarily linear in hours worked as we need to carefully account for incentive costs.

Total output One key quantity in labor markets is total output because a social planner objective is usually interested in high output. ${ }^{14}$ The optimal policy allows for a clear comparison to the outside option in terms of total output. Under a technical assumption on the type distribution $F$ that is a slightly stronger version of logconcavity of $F$, we obtain the following result.

Proposition 7. Let the mapping $x \mapsto f(\exp (x))$ be (strictly) concave on $[\underline{\theta}, \bar{\theta}] \cdot{ }^{15}$ Then, total output under the optimal policy is (strictly) smaller than output under the outside option policy, i.e.

$$
\int_{\theta_{1}}^{\theta_{2}} \theta n^{*}(\theta) d F(\theta)<\int_{\theta_{0}}^{\theta_{2}} \theta \hat{n}(\theta) d F(\theta)
$$

The social planner deliberately reduces output today under the optimal policy. This is somewhat surprising since the social planner incorporates that higher output increases the surplus of matches. However, this result neatly demonstrates the true purpose of STW. The ultimate goal of STW is not to maximize output today, but to retain jobs for the future when the economy recovers. It is indeed optimal to reduce output today because incentivizing matches to work hours that increase output as compared to the outside option is too costly.

\subsection{Comparative Statics}

The optimal policy depends on a number of exogenous parameters that we take as given. We now consider exogenous changes in some parameters to illustrate their implications for the optimal policy. This could also guide policy makers in their decision on how to design STW given the state of the economy.

Value of unemployment. If unemployment benefits increase, we observe that the optimal policy requires the following adjustments.

Corollary 2. If $b$ increases, the share of workers who go into unemployment increases $\left(\theta_{1} \uparrow\right)$ while the share of high productivity matches which employ efficient working hours decreases $\left(\theta_{2} \uparrow\right)$ under the optimal policy.

An increase in $b$ generally leads to higher incentive costs when moving matches from unemployment in the outside option to STW in the optimal policy. In addition, more matches would resolve in favor of unemployment of the worker $\left(\theta_{0} \uparrow\right)$. This results in a shift of optimal STW such that the productivity of the lowest type in STW increases and to maintain incentives, less high productivity types do not select into STW.

A similar statement can be made about a change in the value of reallocation $R$.

Corollary 3. If $R$ increases, the share of matches that are dissolved increases $\left(\theta_{1} \uparrow\right)$ under the optimal policy.

As $R$ increases, dissolving the match becomes more attractive for both the social planner and firms and workers, hence leading to an increase in unemployment $\left(\theta_{1} \uparrow\right)$. However, we cannot conclude in

\footnotetext{
${ }^{13}$ For instance, in Germany, the initial STW subsidy of $60 \%$ of the foregone wage for hours not worked corresponds exactly to the unemployment benefit.

${ }^{14} \mathrm{As}$ is also the case in our model where the social planner seeks to obtain a high surplus which is increasing in total output.

${ }^{15}$ Equivalent expressions for this assumption are that $\theta f(\theta)$ is increasing or that the mapping $e(x)=\mathbb{E}_{x \sim F}[\tilde{x} \mid \tilde{x} \leq x]$ is convex.
} 
which direction the share of high productivity types which are unaffected by the policy moves. The ambiguity arises from the two countering effects that (i) the increase in $R$ makes the outside option more attractive which positively affects incentive costs of STW while (ii) the increase in $\theta_{1}$ negatively affects incentive costs of STW since STW incentive transfers have to be made to a lower share of low productivity types.

Marginal costs of public funds. To further understand the role of marginal costs of public funds, it is instructive to consider the model dynamics in the limit.

Corollary 4. If $\tau \rightarrow 0$, then

- $n^{*}$ converges uniformly to efficient production, i.e. $\lim _{\tau \rightarrow 0} \sup _{\theta \in[\theta, \bar{\theta}]}\left|n^{*}(\theta)-n_{\text {eff }}(\theta)\right|=0$, and

- transfers converge uniformly to $t_{C I}$, i.e $\lim _{\tau \rightarrow 0} \sup _{\theta \in[\underline{\theta}, \bar{\theta}]}\left|t^{*}(\theta)-t_{C I}(\theta)\right|=0$.

An interpretation of the case when payments to matches come at no social cost is that it does not matter to the social planner whether public funds are spent or not. It applies when there is no distortion of the funds raised through taxation. Hence, when transfers are not costly to the social planner, transfers have no disadvantage and are used to incentivize agents to implement the socially efficient working hours.

Corollary 5. If $\tau \rightarrow \infty$, then the optimal policy converges to the transfer minimizing policy. Formally, $n^{*}$ converges to $n^{*}(\theta)=c^{\prime-1}\left(\theta-\frac{F\left(\theta_{2}\right)-F(\theta)}{f(\theta)}\right)$ for $\theta \in\left(\theta_{1}, \theta_{2}\right)$ and to the outside option $\hat{n}$ otherwise.

When the marginal costs of public funds explode, the social planner simply seeks to minimize transfers. Thus, the weighted virtual valuation resembles the virtual valuations known from revenue maximizing mechanisms. It is surprising to some extent that in this case, the social planner does not simply pay unemployment benefits that she committed to in an earlier contractual agreement to all matches who would have chosen it in the outside option. In fact, the optimal mechanism that minimizes transfers also reduces hours of some types above $\theta_{0}$ and compensates them accordingly although transfers are extremely costly. The reason is that this can be used to maintain incentives for types below $\theta_{0}$ to have positive working hours such that the social planner can reduce transfers to those match types.

It is worth highlighting that the cost of public funds is expressed in terms of the cost of total transfers. In particular, this means that we assign equal welfare weights to all matches and do not take issues of inequality into consideration.

\section{Calibration}

In order to assess an optimal STW mechanism quantitatively vis-à-vis an existing job retention policy, we calibrate our model to data on the German labor market before 2008. There are several reasons why we choose this particular period of time.

First, it is important to calibrate the key labor market parameters to a context without any relevant STW scheme. The reason is that in our framework, we only solve for moments of the labor market under the assumption that the planner has already chosen an optimal policy. This is not only an unrealistic assumption but also one which we would like to evaluate with our calibration. The German economy before 2008 provides an ideal scenario, as STW played only a minor role for the country's labor market policy prior to the Great recession of 2009. The period before therefore serves as an ideal candidate to quantitatively match a stationary labor market model without an STW policy. In our theoretical framework, this corresponds to the hypothetical separation and hour decisions which matches may choose as 


\begin{tabular}{lll}
\hline Moment & Value & Corresponding model moment \\
\hline Normalization of hours of active matches & 1 & $\mathbb{E}\left[\hat{n} \mid \theta>\theta_{0}\right]$ \\
Share of firms with less than $5 \%$ adjustment of hours & 0.538 & $\mathbb{E}\left[\mathbb{1}_{\{|\hat{n}-1| \leq 0.05\}} \mid \theta>\theta_{0}\right]$ \\
Separation rate & 0.074 & $F\left(\theta_{0}\right)$ \\
Value of unemployment & 0.387 & $\mathrm{U}$ \\
Normalization of productivity & 1 & $\mathbb{E}[\theta]$ \\
& & \\
Efficient unemployment rate & & \\
\hline Implied efficient separation rate $\left(u^{*}=2 \%\right)$ & 0.012 & $F\left(\theta_{\text {eff }}\right)$ \\
Implied efficient separation rate $\left(u^{*}=4 \%\right)$ & 0.026 & $F\left(\theta_{\text {eff }}\right)$ \\
Implied efficient separation rate $\left(u^{*}=6 \%\right)$ & 0.04 & $F\left(\theta_{\text {eff }}\right)$ \\
\hline
\end{tabular}

Table 1: Overview of moments in the data and corresponding model moments. All values from the data are on an annual basis. To experiment with the strength of the externality, we evaluate our model for several values of a hypothetical efficient unemployment rate $u^{*}$ and calibrate the level of efficient separations in our model accordingly.

an outside option. All our moments, therefore, target the hours $\hat{n}$ which matches choose in the absence of STW.

Second, Germany significantly extended the scope of its STW policy at the onset of the financial crisis in 2009, which allows us to evaluate an important real-world policy vis-à-vis the hypothetical optimal policy of our model.

Third, the same context has been used by Cooper et al. (2017) to match and evaluate a quantitative labor market model of STW. Their model is richer and allows to match more moments of the data as it explicitly models job creation and multi-worker firms. However, similar to other papers in the literature they include STW with the intention to evaluate its effects, but without an optimal design approach such that they cannot answer whether the observed policy was optimally chosen. Importantly for us, they report moments of hour adjustments derived from confidential German firm data which are an important ingredient for our calibration.

Our model is a parsimonious partial equilibrium model in the sense that we do not solve for match creation. Instead, the interpretation of a shock in our model is that a certain fraction of the existing stock of matches separate endogenously. STW attempts to alter the match separation decision. As a first moment, we, therefore, match the parameters of the model without STW such that the separation decision of existing matches corresponds to the observed separation rate into unemployment of $0.6 \%$ monthly (7.4\% yearly) in Germany between 1993 and 2002. Together with a monthly job finding rate for unemployed workers of $5.2 \%$, these values imply a steady state unemployment rate of $10.3 \%$. ${ }^{16}$ The optimal policy in our model further depends on the wedge between efficient and inefficient separations, which quantifies the size of the market failure which the social planner aims to correct. For lack of a concrete estimate for the efficient separation rate in the economy, we evaluate and compare the optimal policy for several levels of a hypothetical "efficient unemployment rate". When holding the monthly

\footnotetext{
${ }^{16}$ We take these estimates from Hartung et al. (2018), who use microdata from the Sample of Integrated Labour Market Biographies (SIAB).
} 


\begin{tabular}{llll}
\hline & Parameter & Description & Estimate \\
\hline Shared parameters & $\phi$ & Curvature of disutility of labor & 6.53 \\
& $A$ & Scale of disutility of labor & 0.152 \\
& $\sigma$ & Variance of productivity shock & 0.378 \\
& $\mu$ & Location parameter of distribution & -0.074 \\
$u^{*}=0.02$ & $R$ & Value of job search & 0.25 \\
$u^{*}=0.04$ & $R$ & & 0.29 \\
$u^{*}=0.06$ & $R$ & & 0.32 \\
\hline
\end{tabular}

Table 2: Parameters of the calibrated model with heta $\sim \operatorname{LogNormal}(\mu, \sigma)$.

job-finding rate constant, these imply different counterfactual efficient separation rates which we then target in our model.

We normalize the model by targeting that average hours $\mathbb{E}[\hat{n}]$ are equal to 1 . The important lever for screening under our optimal policy is the response of hours to financial incentives governed by the curvature of the disutility of labor. As expected, hours are not very responsive in the data. The moment we target is the share of firms that adjust average hours by less than $5 \%$ in a given year, which we take from Cooper et al. (2017). In their data, $53.8 \%$ of firms adjust average hours by less. In our model, this maps into the share of matches with hour adjustments of less than $5 \%$ around the average hours.

We assume a log-normal distribution for the productivity shock and normalize the location such that the expected productivity is 1 . The remaining parameter $\sigma$ governs the variability of $\theta$. Given $\sigma$, the location parameter can be set directly to satisfy $\mathbb{E}[\theta]=1$, greatly reducing the difficulty of the calibration exercise.

Lastly, we calibrate the exogenous value of unemployment which incentivizes match separation at the cost of the public. We assume that this value is equal to the share of production that workers receive in unemployment, which we approximate by the production share of labor times the average net replacement rate of unemployment. The former value is related to the average wage in a competitive labor market while the latter is the average share of the wage that accrues to workers in unemployment. The labor share in Germany averaged 0.63 over 1992-2004, while the average net replacement rate in 2004 was 0.69 . We therefore calibrate the value of unemployment to be $\mathbb{E}[\theta * \hat{n}] * 0.63 * 0.69$. Since we do not solve for a general equilibrium model, this moment choice is arguably the weakest. Specifically, through the static nature of our model, the value of unemployment cannot capture any positive effects of finding a future match. A potential extension would account for match creation to more accurately represent the value of unemployment.

Table 1 provides an overview of the moments in the data and their corresponding definition in our model. After setting $U$ and the location parameter of the distribution directly, there are 4 remaining parameters to calibrate: the curvature and constant of the disutility of labor $\phi$ and A, the value of reallocation and search $R$, as well as the shape parameter of the distribution $\sigma$. We solve for these parameters via a general optimization framework to minimize the distance to the moments. Since the model is just identified, a near perfect match between data and model moments can be achieved. Table 2 reports the corresponding parameter estimates, with separate estimates for $R$ depending on the hypothetical natural unemployment rate $u^{*}$ in our counterfactual scenario. 
Figure 7 shows the numerical solution to our optimal policy problem with calibrated parameters, marginal costs of public funds $1+\tau=1.3$ and a hypothetical natural unemployment rate of $1.2 \%$. Because of adverse selection and screening of the optimal policy, panels $7 \mathrm{a}$ and $7 \mathrm{~b}$ show that a large share of the economy reduces hours by a small amount and is compensated with transfers. Panel 7c shows the change in the observed distribution of hours under an optimal STW policy. Compared to the economy without STW, many more matches work at hours below 1 (the full-time equivalent in the model).

We use our calibrated model to derive counterfactual moments under a hypothetical STW policy that is optimal according to our model. How firmly the social planner would intervene depends on the social costs that she associates with transfers to firms. Depending on the marginal costs of social funds $\tau$ and the strength of the separation inefficiency captured by the implied natural unemployment rate $u^{*}$, the optimal policy differs significantly. We let these parameters vary, with implied natural unemployment rates of $2 \%, 4 \%$ and $6 \%$ respectively and values of $\tau$ of $0.3,0.5$ and 0.7 as estimated in the literature (Kleven and Kreiner, 2006).

Our optimal STW often assigns small hour reductions of $0-5 \%$ and thus includes between $30 \%$ and $87 \%$ of all labor market matches in the policy (see table B.1 in the appendix). This is theoretically justified by the small responsiveness of hours to financial incentives, which we calibrated in order to match the rigidness of labor supply. For practical reasons, however, such a policy is unlikely to be relevant. In order to report reasonable moments to give insights into the practical design of an optimal STW policy, we adjust the definition of STW to matches that reduce hours by 5 percentage points or more relative to the full-time equivalent in our model.

Table 3 summarizes the results of our calibration exercise. According to our model, an optimal STW policy in Germany prior to the Great Recession would have interfered strongly in the labor market and would have reduced the annualized separation rate by at least a fifth, from $7.4 \%$ to $4.8 \%$ to $6.1 \%$. In line with proposition 1 , the optimal separation rate is less than the implied efficient separation rate in all scenarios, since it is too costly to implement production efficient hours.

An important qualitative of the optimal STW policy from our model is the existence of an hours cutoff, i.e. a minimum working time that is still supported by the policy. In our calibrations, matches separate whenever hours fall below $54-72 \%$ of full-time equivalent working hours whereas small reductions occur very frequently. This stands in stark contrast to existing policies, which often stipulate a minimum reduction of working time. In Germany in 2009, for example, STW applications required a reduction of working hours by at least $10 \%$. Of course, there are some reasons outside of our model (e.g. administrative costs) why such a requirement would be necessary.

Overall, the optimal STW reduces hours of participants by little. Even with our adjusted definition of STW, the average reduction of matches in STW is between $9 \%$ and $11 \%$. In comparison, matches that are saved from separation reduce hours by $15-24 \%$ on average. Overall, matches on average receive a transfer corresponding to $24.6 \%$ to $47.7 \%$ of the unemployment benefit $b$.

Perhaps surprisingly, the optimal STW policy exhibits a lot of adverse selection. Between 8.3 and $13.2 \%$ of matches in the economy participate in the policy even though they would not have separated without an intervention. In comparison, the policy only saves between 1 to $2.4 \%$ of matches at risk from separation and as a consequence, the majority of matches in the optimal STW policy do not exhibit any measurable employment effects. This indicates that focusing on the employment effects of STW policies can be uninformative on the welfare effects of these policies, since employment effects may be very small in an optimal setting.

However, the results from our calibrations should be taken with a grain of salt since our model simplifies the economy to a great extent. In particular, we assume that the value of finding a job is homogeneous across matches in the economy and that there are no spill-overs between workers, both of which 


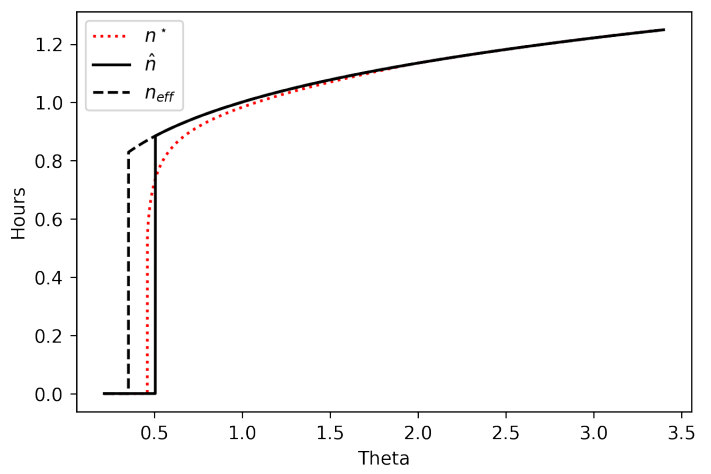

(a) Hours

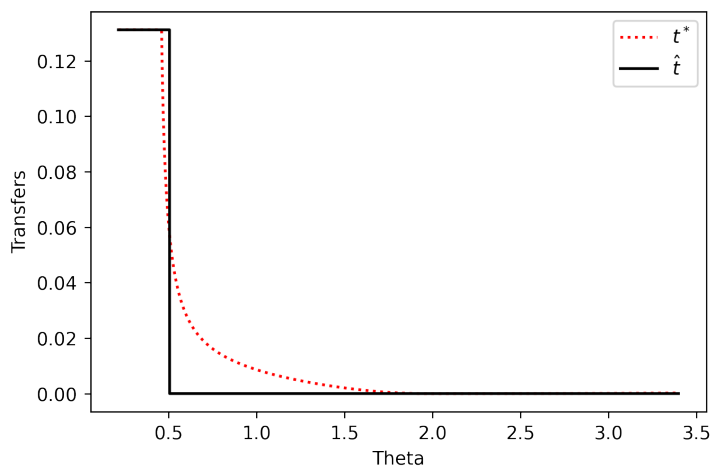

(b) Transfers

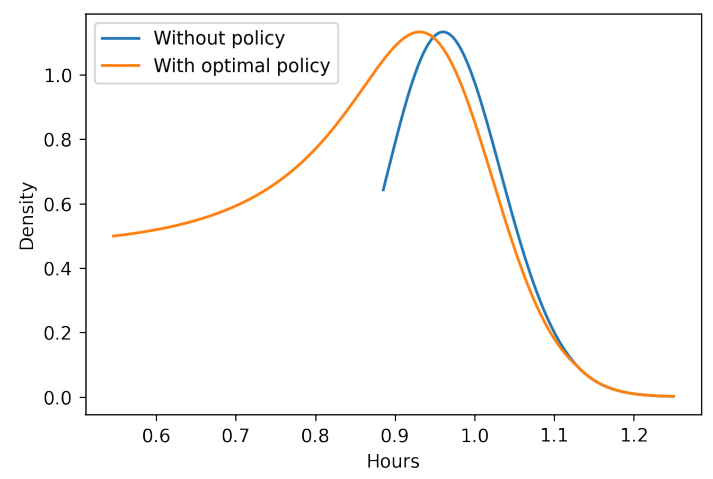

(c) Distribution of hours

Figure 7: Hours, transfers and distribution of hours in a calibrated optimal policy (red, dotted) and without a policy (black, solid). The economy is calibrated to the moments from Table 2, with $u^{*}=0.02$ and $\tau=0.3$

could have a large impact on the quantitative features of an optimal policy. Moreover, we abstract from on-the-job search and instead assume that only separated matches contribute to reallocation.

A final important limitation arises from the fact we matched the curvature of disutility of labor in order to match the rigidity of hours in the economy, which implied a very low elasticity of hours with respect to productivity. Alternatively, the elasticity of existing matches could be low due to bargaining frictions between the firm and worker, whereas a government policy could circumvent these frictions. An ambitious quantification of STW through our model would therefore need to incorporate frictions on the labor market. 


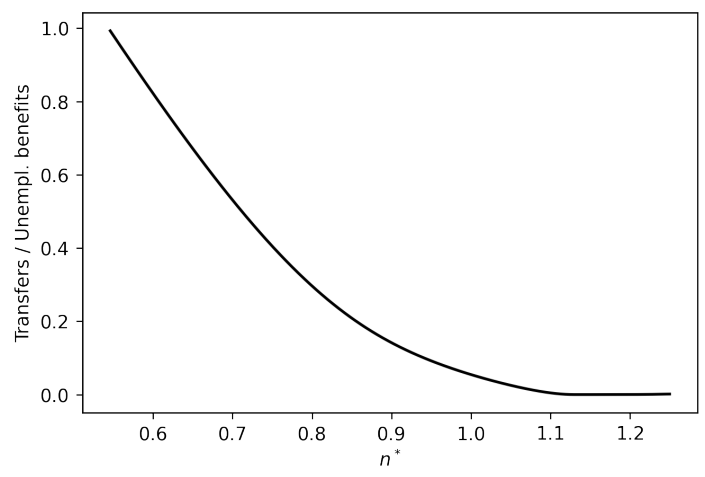

(a)

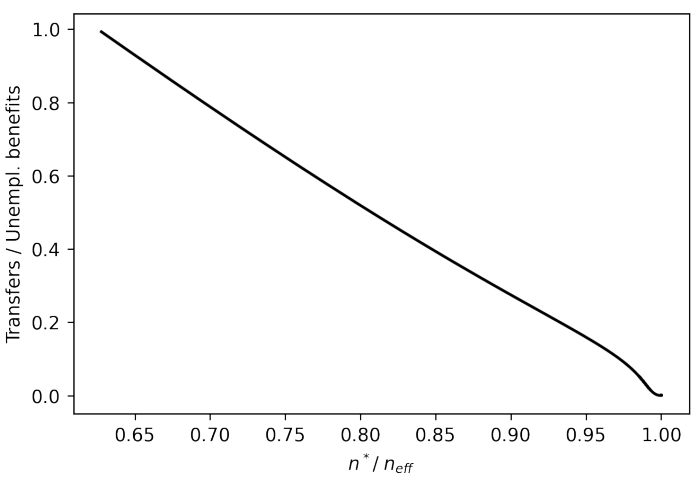

(b)

Figure 8: Transfers relative to unemployment benefit as a function of hours (top) and as a function of hours over first-best hours (bottom). The economy is calibrated to the moments from table 2, with $u^{*}=0.02$ and $\tau=0.3$.

\begin{tabular}{|c|c|c|c|c|c|c|}
\hline \multirow[b]{2}{*}{$\tau$} & \multirow[b]{2}{*}{$u^{*}$} & \multicolumn{5}{|c|}{ Counterfactual moments } \\
\hline & & Separation rate & Hours cutoff & $\begin{array}{l}\text { Av. hours } \\
\text { reduction }\end{array}$ & $\begin{array}{c}\text { Av. hours } \\
\text { reduction } \\
\text { (saved matches) }\end{array}$ & $\begin{array}{l}\text { Av. transfer } \\
\text { relative to UI } \\
\text { benefit }\end{array}$ \\
\hline \multirow[t]{3}{*}{0.3} & 0.02 & 0.048 & 0.55 & 0.1 & 0.21 & $31.3 \%$ \\
\hline & 0.04 & 0.053 & 0.65 & 0.09 & 0.18 & $39.0 \%$ \\
\hline & 0.06 & 0.058 & 0.72 & 0.08 & 0.15 & $47.7 \%$ \\
\hline \multirow[t]{3}{*}{0.5} & 0.02 & 0.054 & 0.54 & 0.1 & 0.23 & $25.7 \%$ \\
\hline & 0.04 & 0.057 & 0.66 & 0.09 & 0.18 & $32.3 \%$ \\
\hline & 0.06 & 0.062 & 0.72 & 0.08 & 0.15 & $39.9 \%$ \\
\hline \multirow[t]{3}{*}{0.7} & 0.02 & 0.055 & 0.55 & 0.11 & 0.24 & $24.6 \%$ \\
\hline & 0.04 & 0.059 & 0.65 & 0.1 & 0.19 & $31.1 \%$ \\
\hline & 0.06 & 0.062 & 0.72 & 0.09 & 0.16 & $39.7 \%$ \\
\hline
\end{tabular}

Table 3: Descriptive moments of the counterfactual optimal short-time work policy. We adjust our definition of short-time work to matches which change hours by more than 0.05 units relative to hours in the outside option. Full-time work is normalized to 1 .

\section{Conclusion}

Recent public debates and the academic literature on STW largely have in common that they provide a positive analysis of STW policies. It is commonly agreed upon that STW schemes in different countries have helped to save jobs especially during times of crisis such as the Great Recession or the Coronavirus pandemic. At the same time, there is a potential deadweight loss from negative selection into STW. Apparent types of adverse selection include (i) viable firm-worker worker matches that select into STW to benefit from government subsidies although they would be maintained without government intervention, (ii) worker layoffs which a social planner would like to prevent, and (iii) take-up of STW that 


\begin{tabular}{ccccc}
\hline & & \multicolumn{3}{c}{ Counterfactual moments } \\
\cline { 3 - 5 }$\tau$ & $u^{*}$ & Adversely selected matches & Share of transfers & Average hour reduction \\
\hline 0.3 & 0.02 & $12.8 \%$ & $21.5 \%$ & 0.08 \\
& 0.04 & $11.3 \%$ & $23.2 \%$ & 0.08 \\
& 0.06 & $8.8 \%$ & $22.7 \%$ & 0.07 \\
0.5 & 0.02 & $13.2 \%$ & $24.4 \%$ & 0.08 \\
& 0.04 & $10.9 \%$ & $25.0 \%$ & 0.08 \\
& 0.06 & $8.3 \%$ & $23.6 \%$ & 0.07 \\
0.7 & 0.02 & $12.6 \%$ & $23.7 \%$ & 0.09 \\
& 0.04 & $10.8 \%$ & $25.2 \%$ & 0.08 \\
& 0.06 & $8.4 \%$ & $24.7 \%$ & 0.07 \\
\hline
\end{tabular}

Table 4: Adverse selection of types under a counter-factual optimal short-time work policy. We adjust our definition of short-time work to matches which change hours by more than 0.05 units relative to hours in the outside option. Full-time work is normalized to 1.

induces inefficient labor hoarding.

In this paper, we have taken a normative mechanism design approach to ask how to design an optimal job retention policy that addresses precisely the aforementioned issues in the presence of firm-worker private information about their need for government support. In particular, we allow for arbitrary coupling of transfers/subsidies to hours worked. Our main result is strikingly simple: A carefully designed STW scheme that respects firm-worker incentives is the optimal policy. STW uses hours worked as a screening tool to avoid unnecessary adverse selection.

Our theoretical results have several practical policy implications. First, even under the optimal STW policy, there exists adverse selection of high productivity matches into STW. Hence, it should not be the ultimate goal of an STW designer to eliminate all adverse selection into STW but rather to balance it well with the positive effects from saving jobs. The structure of our optimal policy suggests that firms and workers should be allowed to even marginally reduce their hours worked. Second, any policy that leads to inefficient layoffs can be improved upon. Well designed STW should set subsidies high enough especially for high hours reductions such that no workers are laid off which would be worth retaining. However, there should be a positive lower bound above zero on hours worked to avoid labor hoarding. The exact optimal design of subsidies and policy targets evidently depends on the precise parameters of the economy and the shape of a shock on productivity. Our calibration of the German economy offers a natural starting point for parameterization.

After its recent widely regarded success, STW policies have most likely been established as an important tool for policymakers in Europe and elsewhere for the years to come. With our theoretical demonstration of STW as a screening mechanism, we hope to facilitate the design of future policies. 


\section{References}

Audenrode, M. A. V. (1994). Short-Time Compensation, Job Security, and Employment Contracts : Evidence from Selected OECD Countries. Journal of Political Economy, 102(1):76-102.

Balleer, A., Gehrke, B., Lechthaler, W., and Merkl, C. (2015). Does Short-time Work save Jobs? A Business Cycle Analysis. Euroropean Economic Review, 84:99-122.

Beal, L., Hill, D., Martin, R., and Hedengren, J. (2018). GEKKO Optimization Suite. Processes, 6(8):106.

Bengea, S. C. and DeCarlo, R. A. (2005). Optimal control of switching systems. Automatica, 41(1):11-27.

Blanchard, O. J. and Tirole, J. (2008). The joint design of unemployment insurance and employment protection: A first pass. Journal of the European Economic Association, 6(1):45-77.

Boeri, T. and Bruecker, H. (2011). Short-time work benefits revisited: some lessons from the Great Recession. Economic Policy, (October):697-765.

Braun, H. and Bruegemann, B. (2017). Welfare Effects of Short-Time Compensation. Tinbergen Institute Discussion Paper, 010/VI.

Burdett, K. and Wright, R. (1989). Unemployment Insurance and Short-Time Compensation: The Effects on Layoffs, Hours per Worker, and Wages. Journal of Political Economy, 97(6):1479-1496.

Cahuc, P., Kramarz, F., and Nevoux, S. (2021). The Heterogeneous Impact of Short-Time Work : From Saved Jobs to Windfall Effects. CEPR Discussion Papers, (DP16168).

Cahuc, P., Nevoux, S., and Cahuc, P. (2018). When Short-Time Work Works. IZA Discussion Paper Series, (11673).

Cesari, L. (2012). Optimization-theory and applications: problems with ordinary differential equations, volume 17.

Chetty, R. (2008). Moral Hazard versus Liquidity and Optimal Unemployment Insurance. Journal of Political Economy, 116(2):173-234.

Christl, M., Poli, S. D., Hufkens, T., Peichl, A., and Ricci, M. (2021). The Role of Short-Time Work and Discretionary Policy Measures in Mitigating the Effects of the Covid-19 Crisis in Germany. CESifo Working Paper Series (9072), (May).

Cooper, R., Meyer, M., and Schott, I. (2017). The Employment and Output Effects of Short-Time Work in Germany. NBER Working Paper, 6(23688):5-9.

Giupponi, G. and Landais, C. (2018). Subsidizing Labor Hoarding in Recessions: the Employment and Welfare Effects of Short Time Work. CEP Discussion Papers, (1585).

Hartung, B., Jung, P., and Kuhn, M. (2018). What Hides Behind the German Labor Market Miracle? Unemployment Insurance Reforms and Labor Market Dynamics. CEP Discussion Papers, (DP13328).

Hijzen, A. and Venn, D. (2011). The Role of Short-Time Work Schemes during the 2008-09 Recession. OECD Social, Employment and Migration Working Papers, (No. 115):46.

Hopenhayn, H. A. and Nicolini, J. P. (1997). Optimal Unemployment Insurance. Journal of Political Economy, 105(2):412-438. 
Huisman, K. J. and Thijssen, J. J. (2020). On the Firm's Option Values of Short-time Work Policies. Mathematics and Financial Economics, 14(2):329-351.

Jullien, B. (1996). L'impact des options extérieures sur les échanges en information asymétrique. Revue économique, 47(3):437-446.

Jullien, B. (2000). Participation Constraints in Adverse Selection Models. Journal of Economic Theory, 93(1):1-47.

Kleven, H. J. and Kreiner, C. T. (2006). The marginal cost of public funds: Hours of work versus labor force participation. Journal of Public Economics, 90(10-11):1955-1973.

Kopp, D. and Siegenthaler, M. (2021). Short-Time Work and Unemployment in and after the Great Recession. Journal of the European Economic Association, 19(4):2283-2321.

Laffont, J.-J. and Tirole, J. (1990). Optimal Bypass and Cream Skimming. American Economic Review, 80(5):1042-1061.

Lewis, T. R. and Sappington, D. E. (1989). Countervailing incentives in agency problems. Journal of Economic Theory, 49(2):294-313.

Martimort, D. and Stole, L. (2020). Participation Constraints in Discontinuous Adverse Selection Models. CEPR Discussion Papers, (DP15251).

Milgrom, P. and Segal, I. (2002). Envelope theorems for arbitrary choice sets. Econometrica, 70(2):583-601.

Mussa, M. and Rosen, S. (1978). Monopoly and product quality. Journal of Economic Theory, 18(2):301-317.

OECD (2020). Job retention schemes during the COVID-19 lockdown and beyond. OECD Policy Responses to Coronavirus (COVID-19), (October):1-23.

Seierstad, A. and Sydsæter, K. (1987). Optimal control theory with economic applications. Elsevier B.V., Amsterdam, The Netherlands.

Sethi, P. S. and Thompson, L. G. (2000). Optimal Control Theory - Applications to Management Science and Economics. Springer, 2nd editio edition.

Stantcheva, S. (2014). Optimal Income Taxation with Adverse Selection in the Labour Market. Review of Economic Studies, 81:1296-1329.

Tilly, J. and Niedermayer, K. (2016). Employment and Welfare Effects of Short-Time Work. Working Paper, (March):1-75.

\section{A Appendix}

\section{A.1 Optimal Policy: Proof of Proposition 2 and Theorem 1}

\section{A.1.1 Notation and Problem Setup}

For convenience throughout the proofs, we define

$$
y:\left\{\begin{array}{l}
{\left[\max \left\{c^{\prime}(0), \underline{\theta}\right\}, \bar{\theta}\right] \longrightarrow \mathbb{R},} \\
\theta \longmapsto\left(c^{\prime}\right)^{-1}(\theta) .
\end{array}\right.
$$


The following Lemma modifies a standard observation in mechanism design to including the variable l.

Lemma 1. Incentive compatibility (IC) for a feasible policy with hours $n(\cdot)$ and inclusion variable $\iota(\theta)$ holds if and only if

$$
\begin{array}{r}
\frac{d S_{P}(\theta)}{d \theta}=\iota(\theta) n(\theta) \text { and } \\
\iota(\theta) n(\theta) \text { is weakly increasing. }
\end{array}
$$

Proof. We let $\tilde{\theta}>\theta$.

Let policy $\{n(\theta), \iota(\theta), t(\theta)\}_{\theta \in[\theta, \bar{\theta}]}$ be feasible and satisfy (IC). Then,

$$
S_{\mathrm{P}}(\theta) \geq \iota(\tilde{\theta}) \theta n(\tilde{\theta})+t(\tilde{\theta})-c(n(\tilde{\theta}))-U+(1-\iota(\tilde{\theta})) R=S_{\mathrm{P}}(\tilde{\theta})+\iota(\tilde{\theta})(\theta-\tilde{\theta}) n(\tilde{\theta})
$$

and

$$
S_{\mathrm{P}}(\tilde{\theta}) \geq \iota(\theta) \tilde{\theta} n(\theta)+t(\theta)-c(n(\theta))-U+(1-\iota(\theta)) R=S_{\mathrm{P}}(\theta)+\iota(\theta)(\tilde{\theta}-\theta) n(\theta)
$$

which, together, implies

$$
\iota(\tilde{\theta}) n(\tilde{\theta}) \geq \frac{S_{\mathrm{P}}(\tilde{\theta})-S_{\mathrm{P}}(\theta)}{\tilde{\theta}-\theta} \geq \iota(\theta) n(\theta) .
$$

This implies (IC'-1) and (IC'-2) follows from letting $\tilde{\theta}$ approach $\theta$.

For the other direction, let $\left(\mathrm{IC}^{\prime}-1\right)$ and $\left(\mathrm{IC}^{\prime}-2\right)$ be true. Then,

$$
S_{P}(\tilde{\theta})-S_{P}(\theta)=\int_{\theta}^{\tilde{\theta}} \iota(s) n(s) d s \geq \int_{\theta}^{\tilde{\theta}} \iota(\theta) n(\theta) d s=(\tilde{\theta}-\theta) \iota(\theta) n(\theta) .
$$

Rearranging, we obtain (14) and (15) can be derived in a similar way.

This is a standard observation in mechanism design and follows from the envelope theorem.

A standard manipulation using (IC), integration by parts allows us to restate the social planner objective as

$$
\begin{aligned}
\mathcal{W}^{\prime} & =\int_{\underline{\theta}}^{\bar{\theta}}(\theta \iota(\theta) n(\theta)+t(\theta)-c(n(\theta))-U+(1-\iota(\theta)) R-(1+\tau) t(\theta) d F(\theta) \\
& =\int_{\underline{\theta}}^{\bar{\theta}}(1+\tau)\left(\iota(\theta) n(\theta)\left(\theta-\frac{\tau}{1+\tau} \frac{1-F(\theta)}{f(\theta)}\right)-c(n(\theta))+(1-\iota(\theta)) R-U\right) d F(\theta)-\tau S_{\mathrm{P}}(\underline{\theta})
\end{aligned}
$$

The problem allows for a statement as an optimal control problem with state constraints. In the following, we mostly follow optimal control solution techniques presented in Seierstad and Sydsæeter (1987) and Sethi and Thompson (2000). The use of inclusion variable $\iota$ as a control variable is also closely related to optimal control problems of switching systems, see e.g. Bengea and DeCarlo (2005). Jullien (2000) introduces an approach to these techniques in models with nontrivial participation constraints and we closely follow his approach.

The state variable in our setting is the match surplus $S_{\mathrm{P}}(\cdot)$. Control variables are hours $n(\cdot)$ and inclusion $\iota(\cdot)$. Their relationship is pinned down via $\left(\mathrm{IC}^{\prime}-1\right)$, i.e. $\dot{S_{\mathrm{P}}}=\imath n$.

The individual rationality constraint (IR) translates into two state constraints. First, we require $S_{\mathrm{P}} \geq 0$ and second we need that $S_{\mathrm{P}} \geq S_{\mathrm{O}}$ on $\left[\theta_{0}, \bar{\theta}\right]$. We split (IR) into two constraints to only deal with continuously differentiable state constraint. The fact that $S_{\mathrm{O}}$ is continuously differentiable on $\left[\theta_{0}, \bar{\theta}\right]$ follows from 


\section{A.1.2 An Optimal Control Problem with State Constraints}

In summary, the optimal control problem states

$$
\begin{aligned}
& \max \left(W^{\prime \prime}\right), \text { s.t. } \quad \dot{S_{\mathrm{P}}}=\imath n, \quad S_{\mathrm{P}} \geq 0, \quad S_{\mathrm{P}} \geq S_{\mathrm{O}} \text { on }\left[\theta_{0}, \bar{\theta}\right], \\
& \iota \in[0,1], n \in[0, \infty) .
\end{aligned}
$$

Note that, as is standard, we have dropped the monotonicity constraint on $\iota n$ and will later verify that the solution to this generalized problem fulfills the constraint.

We can write the Lagrangian as

$$
\begin{aligned}
\mathcal{L}\left(S_{\mathrm{P}}, n, \iota, \varphi, \lambda_{1}, \lambda_{2}, \mu, v_{1}, v_{2}, \theta\right)= & \mathcal{H}\left(S_{\mathrm{P}}, n, \iota, \varphi, \theta\right)+\lambda_{1}\left(S_{\mathrm{P}}-S_{\mathrm{O}}\right)+\lambda_{2} S_{\mathrm{P}}+\mu n, v_{1} \iota+\left(1-v_{2}\right) \iota \\
= & \left(-\tau S_{\mathrm{P}}+(1+\tau)(\theta \iota n-c(n)-U+(1-\iota) R) f(\theta)+\varphi \iota n\right. \\
& +\lambda_{1}\left(S_{\mathrm{P}}-S_{\mathrm{O}}\right)+\lambda_{2} S_{\mathrm{P}}+\mu n+v_{1} \iota+\left(1-v_{2}\right) \iota
\end{aligned}
$$

where $\mathcal{H}$ is the Hamiltonian.

We can state the following necessary conditions for an optimal control.

By the Pontryargin principle, for $\left(S_{\mathrm{P}}^{*}, n^{*}\right)$ to be optimal, it must hold

$$
\begin{aligned}
\dot{S_{\mathrm{P}}}{ }^{*} & =\iota^{*} n^{*} \\
\dot{\varphi} & =-\partial \mathcal{L}\left(S_{\mathrm{P}}^{*}, n^{*}, \iota^{*}, \Delta\right) / \partial S_{\mathrm{P}} \\
\varphi(\bar{\theta}) & =0 \\
\mathcal{H}\left(S_{\mathrm{P}}^{*}, n^{*}, \iota^{*}, \varphi, \theta\right) & \geq \mathcal{H}\left(S_{\mathrm{P}}^{*}, n, \iota, \varphi, \theta\right)
\end{aligned}
$$

for all $(n, \iota) \in[0, \infty) \times[0,1]$.

The transversality condition (21c) states that changing the match surplus of the highest firm type $S_{\mathrm{P}}(\bar{\theta})$ does not change the value of the social planner objective. In addition, we must have $\varphi(\underline{\theta})=-\tau$ because marginally increasing the state variable (i.e. match surplus) at $\underline{\theta}$ will decrease the social planner objective function by a factor $\tau$ given the optimal control $\left(n^{*}, \iota^{*}\right)$. To understand this intuitively, given the optimal control $\left(n^{*}, l^{*}\right)$, the surplus of the lowest type match can only be increased through a transfer which will then have to be made to all higher types as well to maintain incentive compatibility and comes at a $\operatorname{cost} \tau$.

In addition, the Lagrange multipliers must satisfy the complementary slackness conditions

$$
\begin{aligned}
& \lambda_{1} \geq 0, \quad \lambda_{1}\left(S_{\mathrm{P}}-S_{\mathrm{O}}\right)=0 \\
& \lambda_{2} \geq 0, \quad \lambda_{2} S_{\mathrm{P}}=0 \\
& \mu \geq 0, \quad \mu n=0 \\
& v_{1} \geq 0, \quad v_{1} \iota=0 \\
& v_{2} \geq 0, \quad\left(1-v_{2}\right) \iota=0 .
\end{aligned}
$$


There is one more necessary condition to ensure hours $n$ and $\iota$ remain within their defined boundaries.

$$
\left.\frac{\partial \mathcal{L}}{\partial(n, \iota)^{T}}\right|_{(n, l)=\left(n^{*}(\theta), \iota^{*}(\theta)\right)}=0
$$

Importantly, the necessary conditions (21), (22) and (23) are also sufficient conditions for optimality. This follows from Theorem 3, Chapter 5, Seierstad and Sydsæter (1987) since the Hamiltonian is linear in the state variable $S_{\mathrm{P}}$ and therefore weakly concave.

The domain of $n$ can be restricted to a compact set without loss of generality. Any feasible policy with $n(\theta)>n_{\text {eff }}(\bar{\theta})$ can be improved upon by modifying the control variable of hours to $\tilde{n}(\theta) \equiv$ $\min \left\{n(\theta), n_{\text {eff }}(\bar{\theta})\right\}$ while holding control $\iota$ as is. Intuitively, it is strictly dominated to have hours above the efficient level of the most productive match.

With this observation at hand, it suffices to consider a compact domain for the control variables $\iota$ and $n$. Existence of a solution then follows from Neustadt's Existence Theorem for Mayer Problems (see Cesari (2012)).

Lemma 2 (Existence). An optimal policy exists.

\section{A.1.3 Characterization}

The conditions (21), (22), and (23) can be rewritten further to arrive at a solution candidate.

First, the adjoint equation (21b) tells us that $\lambda_{1}+\lambda_{2}-\tau f(\theta)=-\dot{\varphi}$. Let $\lambda \equiv \lambda_{1}+\lambda_{2}$. Define $\tilde{\Lambda}(\theta) \equiv$ $\int_{\underline{\theta}}^{\theta} \lambda(\tilde{\theta}) d F(\tilde{\theta})$. Then, by (21b), we have

$$
\tilde{\Lambda}(\theta)-\tau F(\theta)+m=\tau-\varphi(\theta)
$$

and it is without loss to set $m=\tau^{17}$. Let also $\Lambda(\theta) \equiv \tilde{\Lambda}(\theta) / \tau$.

Then, (21) can be stated as

$$
\left(n^{*}(\theta), \iota^{*}(\theta)\right) \in \underset{(n, \iota) \in[0, \infty) \times[0,1]}{\arg \max } \mathcal{V}(n, \iota)=\left\{\iota n\left(\theta-\frac{\tau}{1+\tau} \frac{\Lambda^{*}(\theta)-F(\theta)}{f(\theta)}\right)-c(n)+(1-\iota) R-U\right\}
$$

with $\Lambda^{*}(\theta)=\left(\int_{\underline{\theta}}^{\theta} \lambda^{*}(\tilde{\theta}) d F(\tilde{\theta})-\varphi(\underline{\theta})\right) / \tau$ for optimal Lagrange multipliers $\lambda_{1}^{*}+\lambda_{2}^{*}=\lambda^{*}$. The initial condition on $\varphi$ and equation (24) ensure that $\Lambda^{*}(\theta) \in[0,1]$ for all $\theta \in[\underline{\theta}, \bar{\theta}]$. This yields the result as stated in Proposition 2.

This result holds although we have a free initial condition, i.e. $S_{\mathrm{P}}(\underline{\theta})$ is not fixed (see Note 9, p. 347, Seierstad and Sydsæter (1987)) with the only additional condition that $\varphi$ is absolutely continuous. This is unlike the standard nonlinear pricing model such as Mussa and Rosen (1978) where the monopolist can always hold the payoff of the lowest type down to the outside option, i.e. to zero. In our case, the participation constraints are involved. The participation constraint could potentially be binding for higher types and jointly with (IC) imply that (IR) does not bind at $\underline{\theta}$, but we later show that this is not the case.

Lemma 3. The participation constraint only binds on at most two intervals $\left[\underline{\theta}, \theta_{1}\right]$ and $\left[\theta_{2}, \bar{\theta}\right]$ with $\underline{\theta} \leq \theta_{1} \leq \theta_{0} \leq$ $\theta_{2} \leq \bar{\theta}$

Proof. Let $\theta<\hat{\theta}$.

Case 1 (interval at the bottom): Let $S_{\mathrm{P}}(\hat{\theta})=S_{\mathrm{O}}(\hat{\theta})=0$ with $\hat{\theta}<\theta_{0}$. Suppose $S_{\mathrm{P}}(\theta)>S_{\mathrm{O}}(\theta)$, i.e.

\footnotetext{
${ }^{17}$ See Note 3, Chapter 5, Seierstad and Sydsæter (1987).
} 
$S_{\mathrm{P}}(\theta)>0$. Then $0<S_{\mathrm{P}}(\theta)=\theta \iota(\theta) n(\theta)+t(\theta)-c(n(\theta))+(1-\iota(\theta))-U \leq \hat{\theta}_{\iota}(\theta) n(\theta)+t(\theta)-c(n(\theta))+$ $(1-\iota(\theta))-U=S_{\mathrm{P}}(\theta \mid \hat{\theta})$ which contradicts incentive compatibility for type $\hat{\theta}$.

Case 2 (interval at the top): Suppose that (IR) binds for $\theta>\theta_{0}$ but not for $\hat{\theta}$ such that $S_{\mathrm{O}}(\theta)=S_{\mathrm{P}}(\theta)$. Now let $\theta^{\dagger}=\inf \left\{\theta \in[\theta, \hat{\theta}] \mid S_{\mathrm{P}}(\theta)-S_{\mathrm{O}}(\theta)>0\right\}$. Then, $S_{\mathrm{P}}\left(\theta^{\dagger}\right)=S_{\mathrm{O}}\left(\theta^{\dagger}\right)$ by continuity of $S_{\mathrm{P}}$ and $S_{\mathrm{O}}$. From the envelope theorem, we also know that $\frac{\partial S_{\mathrm{O}}}{\partial \theta}\left(\theta^{+}\right)=n\left(\theta^{+}\right)$. Together with (IC'-1), it follows that $S_{\mathrm{P}}\left(\theta^{\ddagger}\right) \leq S_{\mathrm{O}}\left(\theta^{\ddagger}\right)$ for $\theta^{\ddagger}$ in some sufficiently small neighborhood around $\theta^{\dagger}$ which contradicts the definition of $\theta^{\dagger}$.

In the following, we will denote the binding intervals as described in Lemma 3 by $\Theta_{1}=\left[\underline{\theta}, \theta_{1}\right]$ and $\Theta_{2}=\left[\theta_{2}, \bar{\theta}\right]$.

Lemma 4. For any optimal policy, the firm participation constraint (IR) must bind for type $\bar{\theta}$ or for $\underline{\theta}$.

Proof. Suppose that policy $\mathcal{P}=\{n(\theta), \iota(\theta), t(\theta)\}_{\theta \in[\underline{\theta}, \bar{\theta}]}$ is optimal, but (IR) is not binding for any match type, i.e. $S_{\mathrm{P}}(\theta)-S_{\mathrm{O}}(\theta)>0$ for all $\theta \in[\underline{\theta}, \bar{\theta}]$. Define $d(\theta):[\underline{\theta}, \bar{\theta}] \longrightarrow \mathbb{R}, \theta \mapsto S_{\mathrm{P}}(\theta)-S_{\mathrm{O}}(\theta)$. Since $S_{\mathrm{P}}(\cdot)$ and $S_{\mathrm{O}}(\cdot)$ are continuous functions, $d$ is a continuous function on a compact interval $[\underline{\theta}, \bar{\theta}]$. Thus, $d$ attains a global minimum which we denote by $d_{\min }$. Consider now the modified mechanism $\{n(\theta), \iota(\theta), t(\theta)-$ $\left.d_{\min }\right\}_{\theta \in[\underline{\theta}, \bar{\theta}]}$ which decreases the transfers to all matches by $d_{\min }$. This policy is still feasible, i.e. $S_{\mathrm{P}}(\theta)-$ $S_{\mathrm{O}}(\theta) \geq 0$, but increases welfare by $\tau d_{\min }$ which leads to a contradiction to the optimality of $\mathcal{P}$. Hence, the participation constraint must bind for some type $\theta \in[\underline{\theta}, \bar{\theta}]$. The claim now follows from Lemma 3.

Lemma 5. For any optimal policy, the firm participation constraint (IR) must bind for $\underline{\theta}$.

Proof. The proof is by contradiction. We assume that a policy for which (IR) does not bind at $\underline{\theta}$ is optimal and construct another policy that leads to a welfare improvement.

Suppose policy $\mathcal{P}=\{n(\theta), \iota(\theta), t(\theta)\}_{\theta \in[\theta, \bar{\theta}]}$ is optimal with $S_{\mathrm{P}}(\underline{\theta})>0$. Lemma 3 and 4 imply that (IR) then binds if and only if $\theta \in[\tilde{\theta}, \bar{\theta}]$ for some $\tilde{\theta} \in\left(\theta_{0}, \bar{\theta}\right]$. It cannot be that $\tilde{\theta}=\theta_{0}$, i.e that (IR) binds for all $\theta \geq \theta_{0}$ but does not bind for $\theta<\theta_{0}$. The reason is that $S_{\mathrm{P}}$ must be monotone increasing by its law of motion (IC'-1, IC'-2) and if $S_{\mathrm{P}}\left(\theta_{0}\right)=S_{\mathrm{O}}\left(\theta_{0}\right)=0$ were true, then $S_{\mathrm{P}}$ would be bounded from above by 0 on $\left[\underline{\theta}, \theta_{0}\right)$ which means that $S_{\mathrm{P}}$ would have to coincide with the outside option $S_{\mathrm{O}}(\theta)=0$ on this interval if $\tilde{\theta}=\theta_{0}$.

Thus, $S_{\mathrm{P}}(\theta)>S_{\mathrm{O}}(\theta)$ for any $\theta<\tilde{\theta}$ with $\tilde{\theta}>\theta_{0}$. This, in particular, implies that for $\theta<\tilde{\theta}$ with $\tilde{\theta}-\theta<$ $\epsilon$ and $\epsilon>0$ sufficiently small, $n(\theta)<\hat{n}(\theta)=n_{\text {eff }}(\theta)$. To see this, note that $S_{\mathrm{P}}(\tilde{\theta})=S_{\mathrm{P}}(\theta)+\int_{\theta}^{\tilde{\theta}} \iota(s) n(s) d s$ and $S_{\mathrm{O}}(\tilde{\theta})=S_{\mathrm{O}}(\theta)+\int_{\theta}^{\tilde{\theta}} \hat{n}(s) d s$. Then, $S_{\mathrm{P}}(\tilde{\theta})=S_{\mathrm{O}}(\tilde{\theta})$ and $S_{\mathrm{P}}(\theta)>S_{\mathrm{O}}(\theta)$ yield $\int_{\theta}^{\tilde{\theta}} l(s) n(s) d s<\int_{\theta}^{\tilde{\theta}} \hat{n}(s) d s$. Since this argument also applies for any $\tilde{\epsilon}<\epsilon$, it follows that $\iota(\theta) n(\theta)<\hat{n}(\theta)=n_{\text {eff }}(\theta)$ for $\theta-\tilde{\theta}<\epsilon$ sufficiently small. Hence, there is underproduction on an interval just below $\tilde{\theta}$.

We now construct a modified policy which saves on transfers while bringing production closer to the efficient level, leading to a welfare improvement. Let $0<\eta<\epsilon$ such that $\delta=\int_{\tilde{\theta}-\eta}^{\theta} n_{\text {eff }}(\theta)-\iota(\theta) n(\theta) d \theta<$ $S_{\mathrm{P}}(\underline{\theta})$. Consider the following policy $\mathcal{P}^{\delta}=\left\{n^{\delta}(\theta), \iota^{\delta}(\theta), t^{\delta}(\theta)\right\}_{\theta \in[\theta, \bar{\theta}]}$ with

$$
n^{\delta}(\theta)= \begin{cases}n(\theta) & \text { if } \theta \leq \tilde{\theta}-\eta \\ n_{\mathrm{eff}}(\theta) & \text { if } \tilde{\theta}-\eta<\theta<\tilde{\theta} \\ n(\theta) & \text { if } \theta \geq \tilde{\theta} .\end{cases}
$$

and

$$
\iota^{\delta}(\theta)= \begin{cases}\iota(\theta) & \text { for } \quad \theta \in[\underline{\theta}, \tilde{\theta}-\eta] \cup[\tilde{\theta}, \bar{\theta}] \\ 1 & \text { else. }\end{cases}
$$


For the policy to be feasible, the envelope condition in integral form then states that transfers are

$$
t^{\delta}(\theta)= \begin{cases}t(\theta)-\delta & \text { if } \theta \leq \tilde{\theta}-\eta \\ c\left(n^{\delta}(\theta)\right)-\theta n^{\delta}(\theta)+\int_{\underline{\theta}}^{\theta} l^{\delta}(s) n^{\delta}(s) d s+U-(1-\iota(\theta)) R+\left(S_{\mathrm{P}}(\underline{\theta})-\delta\right) & \text { if } \tilde{\theta}-\eta<\theta<\tilde{\theta} \\ t(\theta) & \text { if } \theta \geq \tilde{\theta} .\end{cases}
$$

To make a welfare comparison, let us first compare the expenses of the social planner. For $\theta \in(\tilde{\theta}-\eta, \tilde{\theta})$,

$$
\begin{aligned}
t(\theta)-t^{\delta}(\theta) & =\left(c(n(\theta))-\theta \iota(\theta) n(\theta)+\int_{\underline{\theta}}^{\theta} \iota(s) n(s) d s+(1-\iota(\theta)) R S_{\mathrm{P}}(\underline{\theta})\right) \\
& -\left(c\left(n^{\delta}(\theta)\right)-\theta n^{\delta}(\theta)+\int_{\underline{\theta}}^{\theta} \iota^{\delta}(s) n^{\delta}(s) d s+\left(S_{\mathrm{P}}(\underline{\theta})-\delta\right)\right) \\
& =\left(c(n(\theta))-\theta \iota(\theta) n(\theta)-(1-\iota(\theta)) R-c\left(n_{\mathrm{eff}}(\theta)\right)+\theta n_{\mathrm{eff}}(\theta)\right) \\
& +\int_{\tilde{\theta}-\eta}^{\theta} \iota(s) n(s)-n_{\mathrm{eff}}(s) d s+\delta \\
& >0
\end{aligned}
$$

where the last inequality follows from the fact that (31) is positive by the definition of efficient production $n_{\mathrm{eff}}$ and (32) is positive because of the construction of $\delta$ and $n_{\mathrm{eff}}(\cdot)>n(\cdot)$ on $(\tilde{\theta}-\eta, \tilde{\theta})$.

In addition, transfers $t^{\delta}(\cdot)$ and $t(\cdot)$ coincide on $[\tilde{\theta}, \bar{\theta}]$. Hence,

$$
\int_{\underline{\theta}}^{\bar{\theta}} t(\theta)-t^{\delta}(\theta) d F(\theta)=\int_{\tilde{\theta}-\eta}^{\tilde{\theta}} t(\theta)-t^{\delta}(\theta) d F(\theta)+\delta F(\tilde{\theta}-\eta)>0 .
$$

The difference in total welfare is then

$$
\begin{aligned}
\mathcal{W}^{\delta}-\mathcal{W}= & \underbrace{\int_{\tilde{\theta}-\eta}^{\tilde{\theta}} \theta n_{\mathrm{eff}}(\theta)-c\left(n_{\mathrm{eff}}(\theta)\right)-(\theta \iota(\theta) n(\theta)-c(n(\theta))+(1-\iota(\theta)) R) d F(\theta)}_{>0 \text { by efficiency of } n_{\text {eff }}} \\
& +\underbrace{\tau\left(\int_{\underline{\theta}}^{\bar{\theta}} t(\theta)-t^{\delta}(\theta) d F(\theta)\right)}_{>0 \text { by }(34)}>0 .
\end{aligned}
$$

Hence, $\mathcal{P}^{\delta}$ leads to higher welfare than $\mathcal{P}$ which contradicts optimality of $\mathcal{P}$ and concludes the proof.

An important consequence of Lemma 5 is that it is without loss to assume the initial condition $S_{\mathrm{P}}(\underline{\theta})=$ 0 on the state variable. So far, we were studying a program with free initial condition which can now be simplified to fixing the initial value of the state variable.

The further derivation of the optimal policy largely relies on the simple structure as derived in Lemma 3. A useful observation on the intervals where (IR) binds is that the hours $\hat{n}$ chosen absent a social planner intervention are identical to the hours mandated by the optimal policy.

Lemma 6. For any $\theta \in[\underline{\theta}, \bar{\theta}]$, it holds that if $S_{P}(\theta)=S_{O}(\theta)$, then $n^{*}(\theta)=\hat{n}(\theta)$ and $\iota(\theta)=1$ if $\theta \geq \theta_{0}$ and $\iota(\theta)=0$ if $\theta<\theta_{0}$.

Proof. The constraint (IR) can only bind on two intervals $\Theta_{1}$ and $\Theta_{2}$ by Lemma 3.

Let $\theta \in \Theta_{1}$. Then, $\hat{n}=0$. Since $S_{\mathrm{P}}(\theta) \equiv 0$ on $\Theta_{1}$, we must have $\dot{S_{\mathrm{P}}}=0$ and the envelope condition $(\operatorname{IC}-1)$ then requires $\iota^{*}(\theta) n^{*}(\theta)=0$. To see that this implies that $\iota^{*}(\theta)=0$ and $n^{*}(\theta)=0$, first suppose 
that $n^{*}(\theta)>0$ and $\iota^{*}(\theta)=0$. This is in contradiction to the optimality condition (25) since saving costs $c\left(n^{*}(\theta)\right)$ by setting $n(\theta)=0$ is an improvement. Second, suppose that $n^{*}(\theta)=0$ while $\iota^{*}(\theta)>0$. Again, an immediate improvement would be to increase the value of the maximand in (25) by $R$ by setting $\iota^{*}(\theta)=0$.

Now let $\theta \in \Theta_{2}$. The envelope condition on the outside option requires $\dot{S_{\mathrm{O}}}=\hat{n}>0$ on $\Theta_{2}$. Then, together with (25), it must hold that

$$
\iota^{*} n^{*}=\dot{S_{\mathrm{P}}}=\dot{S_{\mathrm{O}}}=\hat{n}
$$

To see that this implies $\iota^{*}=1$ and $n^{*}=1$, suppose that (37) holds true with $\iota^{*}<1, n^{*}>1$. Since $\hat{n}>0$, we must have $\iota^{*} \in(0,1)$. Linearity in $\iota$ of the maximand of the necessary condition (25) requires for $\iota \in(0,1)$ to be optimal that $R=n^{*}\left(\theta-\frac{\tau}{1+\tau} \frac{\Lambda^{*}-F(\theta)}{f(\theta)}\right)$. The value of the maximand is then $R-c\left(n^{*}\right)-U$. A direct improvement would be to set $\iota=0$ and $n=0$ which has a value of $R-U>R-c\left(n^{*}\right)-U$. This is a contradiction to optimality of $\left(\iota^{*}, n^{*}\right)$ which proves the claim.

Lemma 7. For any optimal policy, the participation constraint (IR) must bind for $\bar{\theta}$.

Proof. The proof is by contradiction. Suppose that (IR) does not bind for type $\bar{\theta}$ for an optimal policy

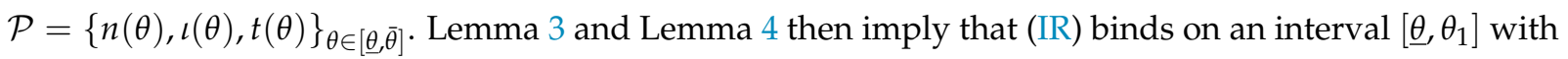
$\theta_{1} \leq \theta_{0}$ but never binds on $\left(\theta_{1}, \bar{\theta}\right]$. We first argue that there must be underproduction, i.e $n<n_{\text {eff }}$, on $\left[\theta_{1}, \bar{\theta}\right)$. Since $\Lambda^{*}(\bar{\theta})=1$ and $\Lambda^{*}$ is constant on an interval where (IR) does not bind, we have $\Lambda^{*}(\theta)=1$ for $\theta \in\left[\theta_{1}, \bar{\theta}\right]$. By the optimality condition (25), for $\theta \in\left(\theta_{1}, \bar{\theta}\right)$, we have $n(\theta)=y\left(\theta-\frac{\tau}{1+\tau} \frac{1-F(\theta)}{f(\theta)}\right)<$ $y(\theta)=n_{\mathrm{eff}}(\theta)$ where the inequality follows from the fact that $c^{\prime}$ is monotone increasing and thus also $\left(c^{\prime}\right)^{-1}$, i.e. $y$. In addition, $n(\bar{\theta})=n_{\text {eff }}(\theta)$. In short, it must hold that

$$
n(\theta) \leq n_{\text {eff }}(\theta) \quad \text { a.e. on }\left[\theta_{1}, \bar{\theta}\right]
$$

On the other hand, if (IR) does not bind for $\bar{\theta}$, this means that $S_{\mathrm{P}}(\bar{\theta})>S_{\mathrm{O}}(\bar{\theta})$. Using the envelope condition for the maximization problem in the outside option and for the policy surplus, we obtain

$$
\int_{\theta_{1}}^{\bar{\theta}} n(\theta) d \theta=S_{\mathrm{P}}(\theta)>S_{\mathrm{O}}\left(\theta_{1}\right)=\int_{\theta_{1}}^{\bar{\theta}} n_{\mathrm{eff}}(\theta) d \theta
$$

which implies that $n_{\text {eff }}>n$ on some set with positive measure. However, this contradicts (38) which concludes the proof.

We now proceed to find the optimal $\Lambda^{*}$ which will fully characterize the optimal policy jointly with (25).

The variable $\Lambda^{*}$ is the critical multiplier. The Lagrange multiplier $\mu$ which ensures that $n \geq 0$ shall always be satisfied when the (IR) constraint is satisfied as well. This leads us to set $\mu \equiv 0$ for now and we will later verify that the constraint $n \geq 0$ is never violated.

Lemma 5 states that $S_{\mathrm{P}}(\underline{\theta})=S_{\mathrm{O}}(\underline{\theta})=0$. Hence, $n^{*}(\underline{\theta})=0$ by Lemma 6 .

Lemma 8. The $(\tau-M V)$ is an increasing function in $\theta$, i.e.

$$
\theta-\frac{\tau}{1+\tau} \frac{\Lambda-F(\theta)}{f(\theta)}
$$

is increasing in $\theta$ for any fixed $\Lambda \in[0,1]$ 
Proof. This follows from the technical assumptions on $F$, i.e. the increasing hazard rate and $f(\theta) / F(\theta)$ is also increasing.

Lemma 9 ( $\iota$-bang-bang). In an optimal policy, $\iota^{*} \equiv 0$ on $\left[\underline{\theta}, \theta_{1}\right]$ and $\iota^{*} \equiv 1$ on $\left[\theta_{1}, \bar{\theta}\right]$.

Proof. We have already argued in the proof of Lemma 6 that $\iota^{*}(\theta)=0$ for all $\theta \in\left[\underline{\theta}, \theta_{1}\right]$ and $\iota^{*}(\theta)=1$ for all $\theta \in\left[\theta_{2}, \bar{\theta}\right]$. Now let $\theta \in\left(\theta_{1}, \theta_{2}\right)$.

First, suppose that $\iota^{*} \in(0,1)$. We can partially repeat an argument from Lemma 6 . Linearity in $\iota$ of the maximand of the necessary condition (25) requires for $\iota \in(0,1)$ to be optimal that $R=n^{*}\left(\theta-\frac{\tau}{1+\tau} \frac{\Lambda^{*}-F(\theta)}{f(\theta)}\right)$. The value of the maximand is then $R-c\left(n^{*}\right)-U$. A direct improvement would be to set $\iota=0$ and $n=0$ which has a value of $R-U>R-c\left(n^{*}\right)-U$. This is a contradiction to optimality of $\left(\iota^{*}, n^{*}\right)$.

Second, suppose that $\iota^{*}(\theta)=0$. Then, as is outlined in the first part of the proof of Lemma 6, we have $n^{*}(\theta)=0$. Next, we argue that we then have $n^{*} \iota^{*}=0$ on $\left(\theta_{1}, \theta\right)$. If not, there would be $\tilde{\theta} \in\left(\theta_{1}, \theta\right)$ with $n^{*}(\tilde{\theta}), \iota^{*}(\tilde{\theta})>0$ such that $R-U \leq \iota^{*}(\tilde{\theta}) n^{*}(\tilde{\theta})\left(\tilde{\theta}-\frac{\tau}{1+\tau} \frac{\Lambda^{*}(\tilde{\theta})-F(\tilde{\theta})}{f(\tilde{\theta})}\right)-c\left(n^{*}(\tilde{\theta})\right)+\left(1-\iota^{*}(\tilde{\theta})\right)-U<$ $\iota^{*}(\tilde{\theta}) n^{*}(\tilde{\theta})\left(\theta-\frac{\tau}{1+\tau} \frac{\Lambda^{*}(\theta)-F(\theta)}{f(\theta)}\right)-c\left(n^{*}(\tilde{\theta})\right)+\left(1-\iota^{*}(\tilde{\theta})\right)-U$. Hence, $\left(n^{*}(\tilde{\theta}), \iota^{*}(\tilde{\theta})\right)$ would be an improvement for type $\theta$ over $\left(n^{*}(\theta), \iota^{*}(\theta)\right)=(0,0)$ in (25), a contradiction. Therefore, $n^{*} \iota^{*} \equiv 0$ on $[\underline{\theta}, \theta]$ and by $\left(\mathrm{IC}^{\prime}-1\right), S_{\mathrm{P}} \equiv 0$ on $[\underline{\theta}, \theta]$, which contradicts that $S_{\mathrm{P}}>S_{\mathrm{O}}$ on $\left(\theta_{1}, \theta\right)$.

Summary of Necessary and Sufficient Conditions. Thus far, we have derived conditions of optimality for optimization problem that simplify its structure. The state constraints bind on two intervals $\left[\underline{\theta}, \theta_{1}\right]$ and $\left[\theta_{2}, \bar{\theta}\right]$ where $\theta_{1} \leq \theta_{2}$. Condition 22 implies that $\Lambda^{*}(\cdot)$ is constant on $\left(\theta_{1}, \theta_{2}\right)$. Together with (25), the optimization problem reduces to find optimal threshold values $\theta_{1}, \theta_{2}$ and a constant $\Lambda_{\left(\theta_{1}, \theta_{2}\right)}^{*}$. Then,

$$
\Lambda^{*}(\theta)= \begin{cases}\left(\theta-\theta_{\text {eff }}\right) f(\theta)+F(\theta) & \text { for } \theta=\theta_{1} \\ \Lambda_{\left(\theta_{1}, \theta_{2}\right)}^{*} & \text { for } \theta_{1}<\theta<\theta_{2} \\ F(\theta) & \text { for } \theta \geq \theta_{2}\end{cases}
$$

In addition, $\Lambda^{*}$ needs to satisfy

$$
\Lambda^{*}(\theta)>\left(\theta-\theta_{\text {eff }}\right) f(\theta)+F(\theta) \quad \text { for } \theta<\theta_{1} \text {. }
$$

Hence, $\Lambda^{*}$ is not uniquely defined on $\left[\underline{\theta}, \theta_{1}\right]$, but all admissible values induce the same optimal policy.

However, we can further characterize the threshold values $\theta_{1}$ and $\theta_{2}$. By Berge's maximum theorem, $\mathcal{V}\left(n^{*}, \iota^{*}\right)(\theta)$ from (25) must be continuous in $\theta$. This is only possible if $\Lambda^{*}$ is continuous at $\theta_{1}$ and $\theta_{2}$. Hence,

$$
F\left(\theta_{2}\right)=\Lambda_{\left(\theta_{1}, \theta_{2}\right)}^{*} \text { and }\left(\theta_{1}-\theta_{\text {eff }}\right) f\left(\theta_{1}\right)+F\left(\theta_{1}\right)=\Lambda_{\left(\theta_{1}, \theta_{2}\right)}^{*} .
$$

For given $\Lambda_{\left(\theta_{1}, \theta_{2}\right)}^{*}$, an optimal policy then takes the form

$$
\begin{gathered}
n^{*}(\theta)= \begin{cases}0 & \text { if } \theta \leq \theta_{1} \\
y\left(\theta-\frac{\tau}{1+\tau} \frac{\Lambda_{\left(\theta_{1}, \theta_{2}\right)}^{*}-F(\theta)}{f(\theta)}\right) & \text { if } \theta_{1}<\theta<\theta_{2} \\
y(\theta) & \text { if } \theta \geq \theta_{2},\end{cases} \\
\iota^{*}(\theta)= \begin{cases}0 & \text { if } \theta<\theta_{1} \\
1 & \text { if } \theta \geq \theta_{1}\end{cases}
\end{gathered}
$$


with Lagrange multipliers (as derived from (23)) are

$$
\begin{aligned}
& \varphi^{*}(\theta)=\tau\left(F(\theta)-\Lambda_{\left(\theta_{1}, \theta_{2}\right)}^{*}(\theta)\right), \\
& \lambda_{1}^{*}(\theta)=\mathbb{1}_{\left\{\theta \in\left[\theta_{1}, \theta_{1}\right]\right\}}(\theta) \cdot \frac{\partial \Lambda^{*}}{\partial \theta}, \\
& \lambda_{2}^{*}(\theta)=\mathbb{1}_{\left\{\theta \in\left[\theta_{2}, \bar{\theta}\right]\right\}}(\theta) \cdot \frac{\partial \Lambda^{*}}{\partial \theta}, \\
& \nu_{1}^{*}(\theta)=\mathbb{1}_{\left\{\theta \in\left[\theta, \theta_{1}\right]\right\}}(\theta) \cdot[(1+\tau) R f(\theta)], \\
& v_{2}^{*}(\theta)=\mathbb{1}_{\left\{\theta \in\left[\theta_{2}, \bar{\theta}\right]\right\}}(\theta) \cdot\left[(1+\tau)\left(\theta n^{*}(\theta)-R\right) f(\theta)+\varphi^{*}(\theta) n^{*}(\theta)\right], \\
& \mu^{*}(\theta)=\mathbb{1}_{\left\{\theta \in\left[\theta_{1}, \theta_{1}\right]\right\}}(\theta) \cdot(1+\tau) c\left(n^{*}(\theta)\right) f(\theta) .
\end{aligned}
$$

With the characterization at hand, we can restate problem (17) as

$$
\begin{aligned}
\max _{\Lambda \in[\underline{\Lambda}, \bar{\Lambda}]} \mathcal{W}^{\prime \prime \prime} & =\left\{\int_{\underline{\theta}}^{\theta_{1}} R-\operatorname{UdF}(\theta)\right. \\
& +\int_{\theta_{1}}^{F^{-1}(\theta)} y\left(\theta-\frac{\tau}{1+\tau} \frac{\Lambda-F(\theta)}{f(\theta)}\right)\left[\theta-\frac{\tau}{1+\tau} \frac{\Lambda-F(\theta)}{f(\theta)}\right]-c\left(y\left(\theta-\frac{\tau}{1+\tau} \frac{\Lambda-F(\theta)}{f(\theta)}\right)\right)-U d F(\theta) \\
& \left.+\int_{F^{-1}(\Lambda)}^{\bar{\theta}} \theta y(\theta)-c(y(\theta))-\operatorname{UdF}(\theta)\right\}
\end{aligned}
$$

s.t. (i) $\theta_{1}$ is the root of

$$
\xi_{\Lambda}(\theta) \equiv y\left(\theta-\frac{\tau}{1+\tau} \frac{\Lambda-F(\theta)}{f(\theta)}\right)\left[\theta-\frac{\tau}{1+\tau} \frac{\Lambda-F(\theta)}{f(\theta)}\right]-c\left(y\left(\theta-\frac{\tau}{1+\tau} \frac{\Lambda-F(\theta)}{f(\theta)}\right)\right)-R
$$

if $\Lambda<1$ while $\theta_{1}=\theta_{0}$ if $\Lambda=1$,

(ii) $\quad \int_{\theta_{1}}^{F^{-1}(\Lambda)} y\left(\theta-\frac{\tau}{1+\tau} \frac{\Lambda-F(\theta)}{f(\theta)}\right) d \theta=S_{\mathrm{O}}\left(F^{-1}(\Lambda)\right)$,

(iii) $\quad \underline{\Lambda}=F\left(\theta_{0}\right) \quad$ and $\quad \bar{\Lambda}=\min \left\{F\left(\theta_{0}\right)+f\left(\theta_{0}\right)\left(\theta_{0}-\theta_{\text {eff }}\right), 1\right\}$.

In essence, this modified optimization problem is constructed by plugging in the necessary functional form of optimal controls $\left(n^{*}, \iota^{*}\right)$ as in (44) and (45) into the objective (W") and adjusting the constraints accordingly. It captures the fact that the problem reduces to finding an optimal value $\Lambda_{\left(\theta_{1}, \theta_{2}\right)}^{*}$ that governs where the state constraints bind.

We first simplify condition (52f). The function $\tilde{\zeta}(x) \equiv y(x) x-c(y(x))-R$ has at most one root since $\tilde{\xi}^{\prime}(x)=y(x)>0$. By definition of $\theta_{\text {eff }}$, the root exists and is implicitly defined by $\tilde{\xi}^{\prime}\left(\theta_{\text {eff }}\right)=0$. Hence, any root of $\xi_{\Lambda}(\theta)$ needs to satisfy $\theta-\frac{\tau}{1+\tau} \frac{\Lambda-F(\theta)}{f(\theta)}=\theta_{\text {eff. }}$. If $F\left(\theta_{0}\right)+f\left(\theta_{0}\right)\left(\theta_{0}-\theta_{\text {eff }}\right) \leq 1$, then $\theta_{1} \in\left[\theta_{\text {eff }}, \theta_{0}\right]$. In short, (52f) can be rewritten to

$$
\theta_{1} \begin{cases}\text { solves } \theta_{1}-\frac{\tau}{1+\tau} \frac{\Lambda-F\left(\theta_{1}\right)}{f\left(\theta_{1}\right)}=\theta_{\text {eff }} & \text { if } \Lambda<1 \\ =\theta_{0} & \text { if } \Lambda=1\end{cases}
$$

Let $\chi(\Lambda) \equiv \int_{\theta_{1}}^{F^{-1}(\Lambda)} y\left(\theta-\frac{\tau}{1+\tau} \frac{\Lambda-F(\theta)}{f(\theta)}\right) d \theta-S_{\mathrm{O}}\left(F^{-1}(\Lambda)\right)$.

Lemma 10. There exists a unique $\Lambda^{\prime \prime \prime} \in[\underline{\Lambda}, \bar{\Lambda}]$ that satisfies (52f) and (52g). 
Proof. Applying the Leibniz integral rule, we have

$$
\begin{aligned}
\chi^{\prime}(\Lambda)= & \frac{d}{d \Lambda}\left(F^{-1}(\Lambda)\right) \cdot y\left(F^{-1}(\Lambda)\right)-\frac{d \theta_{1}}{d \Lambda} \cdot y\left(\theta_{1}-\frac{\tau}{1+\tau} \frac{\Lambda-F\left(\theta_{1}\right)}{f\left(\theta_{1}\right.}\right) \\
& +\int_{\theta_{1}}^{F^{-1}(\Lambda)} \frac{d}{d \Lambda} y\left(\theta-\frac{\tau}{1+\tau} \frac{\Lambda-F(\theta)}{f(\theta)}\right) d F(\theta)-\frac{d}{d \Lambda}\left(F^{-1}(\Lambda)\right) \cdot y\left(F^{-1}(\Lambda)\right) \\
= & -\frac{d \theta_{1}}{d \Lambda} \cdot y\left(\theta_{1}-\frac{\tau}{1+\tau} \frac{\Lambda-F\left(\theta_{1}\right)}{f\left(\theta_{1}\right.}\right)+\int_{\theta_{1}}^{F^{-1}(\Lambda)} \frac{d}{d \Lambda} y\left(\theta-\frac{\tau}{1+\tau} \frac{\Lambda-F(\theta)}{f(\theta)}\right) d F(\theta)
\end{aligned}
$$

Define $h_{\tau}(\theta, \Lambda)=\theta-\frac{\tau}{1+\tau} \frac{\Lambda-F(\theta)}{f(\theta)}$. We now decompose (56). Total differentiation of $h_{\tau}\left(\theta_{1}, \Lambda\right)$ w.r.t. $\Lambda$ has to equal 0 in order to maintain that $h_{\tau}\left(\theta_{1}, \Lambda\right)=\theta_{\text {eff }}$ as required by (53). Then,

$$
\frac{d \theta_{1}}{d \Lambda}=\left[\frac{\partial h_{\tau}\left(\theta_{1}, \Lambda\right)}{\partial \theta_{1}}\right]^{-1} \cdot\left[\frac{\partial h_{\tau}\left(\theta_{1}, \Lambda\right)}{\partial \Lambda}\right]=\left[\frac{\partial h_{\tau}\left(\theta_{1}, \Lambda\right)}{\partial \theta_{1}}\right]^{-1} \cdot\left[\frac{\tau}{1+\tau} \frac{1}{f\left(\theta_{1}\right)}\right]>0
$$

where $\frac{\partial h_{\tau}\left(\theta_{1}, \Lambda\right)}{\partial \theta_{1}}>0$ is a consequence of Lemma 8. In addition, an application of the inverse function theorem to $y^{\prime}$ yields

$$
\begin{aligned}
\frac{d}{d \Lambda} y\left(\theta-\frac{\tau}{1+\tau} \frac{\Lambda-F(\theta)}{f(\theta)}\right) & =\left[-\frac{1}{f(\theta)}\right] y^{\prime}\left(\theta-\frac{\tau}{1+\tau} \frac{\Lambda-F(\theta)}{f(\theta)}\right) \\
& =\left[-\frac{1}{f(\theta)}\right] c^{\prime \prime}\left(y\left(\theta-\frac{\tau}{1+\tau} \frac{\Lambda-F(\theta)}{f(\theta)}\right)\right)<0
\end{aligned}
$$

Hence, $\chi^{\prime}(\Lambda)<0$.

We proceed to calculate the boundary values $\chi(\underline{\Lambda})$ and $\chi(\bar{\Lambda})$. We have

$$
\chi(\underline{\Lambda})=\int_{\theta_{1}}^{\theta_{0}} y\left(\theta-\frac{\tau}{1+\tau} \frac{F\left(\theta_{0}\right)-F(\theta)}{f(\theta)}\right) d \theta \geq 0
$$

Also, $\bar{\Lambda}=F\left(\theta_{0}\right)+f\left(\theta_{0}\right)\left(\theta_{0}-\theta_{\text {eff }}\right) \leq 1$, then $\theta_{1}=\theta_{0}$ as is also the case if $\bar{\Lambda}=1$. Hence,

$$
\chi(\bar{\Lambda})=\int_{\theta_{0}}^{\bar{\theta}}\left(y\left(\theta-\frac{\tau}{1+\tau} \frac{\bar{\Lambda}-F(\theta)}{f(\theta)}\right)-y(\theta)\right) d \theta \leq 0
$$

since $\theta-\frac{\tau}{1+\tau} \frac{\bar{\Lambda}-F(\theta)}{f(\theta)} \leq \theta$. The result follows from the Poincaré-Hopf index theorem.

As $\Lambda^{*}$ remains to be the only candidate that satisfies the constraint $(52 \mathrm{~g})$, it is also the unique solution to the program (52). It is left to formally argue that the solution space of (52) coincides with the solution space of (17).

A solution to (17) consists of controls $\left(n^{*}(\cdot), \iota(\cdot)\right)_{\{[\theta, \bar{\theta}]\}}$ and constant $\Lambda_{\left(\theta_{1}, \theta_{2}\right)}^{*}$ that induces Lagrange multipliers (41) and (46).

We denote the set of solutions by $\Omega^{\prime \prime}=\left\{\left(n^{*}, \iota^{*}, \Lambda_{\left(\theta_{1}, \theta_{2}\right)}^{*}\right) \mid(41),(44),(45),(43)\right.$ are satisfied $\}$.

Besides, a solution to (52) is constant $\Lambda^{\prime \prime \prime}$ and we denote the set of solutions by $\Omega^{\prime \prime \prime}$. This is a singleton as argued before. 
Lemma 11. The mapping

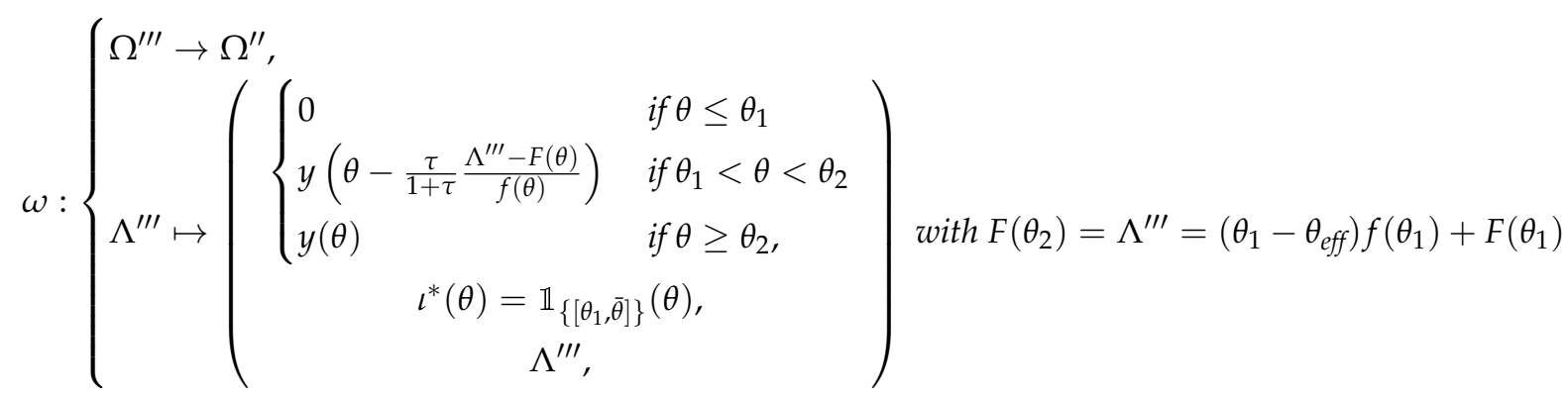

is a bijection.

Proof. Consider a solution $\left({ }^{*}, \iota^{*}\right)$ to (17) which take the form as in (44) and (45) for $\Lambda_{\left(\theta_{1}, \theta_{2}\right)}^{*}$. Hence, the mapping is well defined. (IR) binds above $\theta_{2}=F^{-1}\left(\Lambda_{\left(\theta_{1}, \theta_{2}\right)}^{*}\right)$ which implies that (52g) applies for $\Lambda_{\left(\theta_{1}, \theta_{2}\right)}^{*}$. In addition, $\Lambda_{\left(\theta_{1}, \theta_{2}\right)}^{*} \in[\underline{\Lambda}, \bar{\Lambda}]$. If $\theta<F\left(\theta_{0}\right)$, then $\theta_{2}<\theta_{0}$ and if $\bar{\Lambda}>F\left(\theta_{0}\right)+f\left(\theta_{0}\right)\left(\theta_{0}-\theta_{\text {eff }}\right)$, then $\theta_{1}>\theta_{0}$ which contradicts Lemma 3 in either case. In addition, we know $\Lambda_{\left(\theta_{1}, \theta_{2}\right)}^{*} \leq 1$. Hence, $\Lambda_{\left(\theta_{1}, \theta_{2}\right)}^{*}$ is an admissible in the program (52). We showed in Lemma 10 that it is then also a solution to (52).

Now consider a solution $\Lambda^{\prime \prime \prime} \in \Omega^{\prime \prime \prime}$. The functions $n^{*}$ and $\iota^{*}$ that $\omega\left(\Lambda^{\prime \prime \prime}\right)$ maps to satisfy (25) (or equivalently (21) if we assume that Lagrange multipliers are constructed as in (46)). In addition, the surplus condition (52g) implies that $S_{\mathrm{P}}\left(F^{-1}\left(\Lambda^{\prime \prime \prime}\right)\right)=S_{\mathrm{O}}\left(F^{-1}\left(\Lambda^{\prime \prime \prime}\right)\right)$. Since $\Lambda^{*}$ is constant on $\left(F^{-1}\left(\Lambda_{\left(\theta_{1}, \theta_{2}\right.}^{*}\right), \bar{\theta}\right]$ by construction in (41), the complementary slackness conditions (22) are satisfied. Thus, the necessary (and also sufficient) conditions for optimality are satisfied and $\omega\left(\Lambda^{\prime \prime \prime}\right)$ indeed induces a solution for (17).

Note that it is immediate from (44), (45) jointly with (41) and Lemma 8 that $n^{*} \iota^{*}$ is monotone increasing in $\theta$ so that it was without loss to drop the monotonicity constraint.

Lemma 11 states that it is sufficient to find solution to the simplified problem (52). The surplus condition $(52 \mathrm{~g})$ in (52) therefore allows us to fully characterize the optimal solution. This concludes the proof of Theorem 1.

\section{A.2 Proofs of other results}

\section{Proof of Proposition 1.}

Proof. Suppose policy $\mathcal{P}_{\text {eff }}=\{n(\theta), \iota(\theta), t(\theta)\}_{\theta \in[\theta, \bar{\theta}]}$ is optimal with

$$
n(\theta)= \begin{cases}y(\theta) & \text { if } \theta \geq \theta_{\text {eff }} \\ 0 & \text { else }\end{cases}
$$

and $\iota(\theta)=\iota_{\text {eff }}(\theta)$. A feasible policy has to satisfy (IC) which by Lemma 1 implies that transfers can be written as

$$
t(\theta)=c(n(\theta))-\theta \iota(\theta) n(\theta)+\int_{\underline{\theta}}^{\theta} \iota(s) n(s) d s+S_{\mathrm{P}}(\underline{\theta})+U-(1-\iota(\theta)) R .
$$

Plugging in from (63), we have $t(\theta) \equiv S(\underline{\theta})+U-R=S(\underline{\theta})+b$ for $\theta \in\left[\underline{\theta}, \theta_{\text {eff }}\right]$. For $\theta \in\left(\theta_{\text {eff }}, \bar{\theta}\right]$,

$$
t^{\prime}(\theta)=c^{\prime}(y(\theta)) y^{\prime}(\theta)-y^{\prime}(\theta) \theta-y(\theta)+\frac{d}{d \theta} \int_{\underline{\theta}}^{\theta} n(s) d s=\theta y^{\prime}(\theta)-y^{\prime}(\theta) \theta=0 .
$$


Hence, $t(\theta) \equiv S_{\mathrm{P}}(\underline{\theta})+b$ a.e. and for (IR) to be binding for some type as required by Lemma 4 , set $S(\underline{\theta})=0$ such that $t(\theta) \equiv b$.

Now consider the following alternative policy $\mathcal{P}_{\epsilon}=\left\{n^{\epsilon}(\theta), \iota^{\epsilon}(\theta), t^{\epsilon}(\theta)\right\}_{\theta \in[\underline{\theta}, \bar{\theta}]}$ with

$$
n^{\epsilon}(\theta)= \begin{cases}y(\theta) & \text { if } \theta \geq \theta_{\text {eff }}+\epsilon \\ 0 & \text { else }\end{cases}
$$

$\iota^{\epsilon}(\theta)=\mathbb{1}_{\left\{\theta \geq \theta_{\text {eff }}+\epsilon\right\}}(\theta)$ and

$$
t^{\epsilon}(\theta)= \begin{cases}b-C_{\epsilon} & \text { if } \theta \geq \theta_{\text {eff }}+\epsilon \\ b & \text { else }\end{cases}
$$

where $C_{\epsilon}=\left(\theta_{\mathrm{eff}}+\epsilon\right) y\left(\theta_{\mathrm{eff}}+\epsilon\right)-c\left(y\left(\theta_{\mathrm{eff}}+\epsilon\right)\right)-R$ for some small $\epsilon>0$. It is immediate from the definition of $\theta_{\text {eff }}$ that $C_{\epsilon}>0$ for small $\epsilon>0$. This policy is feasible as can be seen from the fact that transfers are chosen in a way just so that (IC) is fulfilled.

Total welfare for the efficient production implementation is

$$
\mathcal{W}_{\text {eff }}=\int_{\theta_{\text {eff }}}^{\bar{\theta}} \theta y(\theta)-c(y(\theta)) d F(\theta)-\tau b+F\left(\theta_{\text {eff }}\right) R
$$

and total welfare of policy $\mathcal{P}_{\epsilon}$ is

$$
\mathcal{W}_{\epsilon}=\int_{\theta_{\mathrm{eff}}+\epsilon}^{\bar{\theta}} \theta y(\theta)-c(y(\theta)) d F(\theta)-\tau b+F\left(\theta_{\mathrm{eff}}+\epsilon\right) R+\tau\left[1-F\left(\theta_{\mathrm{eff}}+\epsilon\right)\right] C_{\epsilon}
$$

Comparing total welfare then gives

$$
\begin{aligned}
\mathcal{W}_{\epsilon}-\mathcal{W}_{\text {eff }} & =\tau\left[1-F\left(\theta_{\text {eff }}+\epsilon\right)\right] C_{\epsilon}+\left(F\left(\theta_{\text {eff }}+\epsilon\right)-F\left(\theta_{\text {eff }}\right)\right) R-\underbrace{\int_{\theta_{\text {eff }}+\epsilon}^{\theta} \theta y(\theta)-c(y(\theta)) d F(\theta)}_{\leq\left(\theta_{\text {eff }}+\epsilon\right) y\left(\theta_{\text {eff }}+\epsilon\right)-c\left(y\left(\theta_{\text {eff }}+\epsilon\right)\right)\left(F\left(\theta_{\text {eff }}+\epsilon\right)-F(\theta)\right)} \\
& \geq[\underbrace{\tau\left[1-F\left(\theta_{\text {eff }}+\epsilon\right)\right]}_{\stackrel{\epsilon \rightarrow 0}{\tau} \tau\left(1-F\left(\theta_{\text {eff }}\right)\right)}-\underbrace{\left(F\left(\theta_{\text {eff }}+\epsilon\right)-F(\theta)\right)}_{\stackrel{\epsilon \rightarrow 0}{\longrightarrow} 0}] \underbrace{C_{\epsilon}}_{>0 \text { for } \epsilon>0}
\end{aligned}
$$

for sufficiently small $\epsilon$. This shows that policy $\mathcal{P}_{\epsilon}$ is an improvement over policy $\mathcal{P}_{\text {eff }}$ and that concludes the proof.

\section{Proof of Proposition 3.}

Proof. Consider hours $n^{*}(\cdot)$ in an optimal policy. From Theorem 1 , we see immediately that $n^{*}(\theta)=$ $n_{\text {eff }}(\theta)$ for $\theta \in\left[\underline{\theta}, \theta_{1}\right] \cup\left[\theta_{2}, \bar{\theta}\right]$. For $\theta \in\left(\theta_{1}, \theta_{2}\right)$, where $\iota^{*}(\cdot)=1$, we have $F\left(\theta_{2}\right)>F(\theta)$ and therefore $\theta>\theta-\frac{\tau}{1+\tau} \frac{F\left(\theta_{2}\right)-F(\theta)}{f(\theta)}$. The result follows from the fact that $c^{\prime-1}$ is monotone increasing, (6) and $n_{\mathrm{eff}}(\theta)=$ $c^{\prime-1}(\theta)$.

\section{Proof of Proposition 5.}

Proof. This is a direct consequence from the fact that $\theta_{2}>\theta_{1}$ such that $\Lambda=F\left(\theta_{2}\right)>F\left(\theta_{1}\right)$ and condition (8). 


\section{Proof of Corollary 1.}

Proof. If $b=0$, then $\theta_{0}=\theta_{\text {eff }}$.

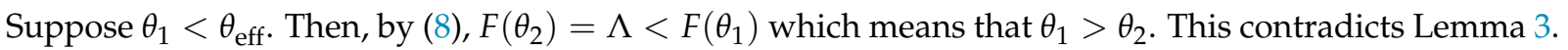
Hence, $\theta_{1}=\theta_{\text {eff }}=\theta_{0}$ and $F\left(\theta_{1}\right)=\Lambda=F\left(\theta_{2}\right)$. With $\theta_{1}=\theta_{2}$, the interval with STW is at most a singleton of measure zero and the outside option binds almost everywhere.

\section{Proof of Proposition 7.}

Proof. (Strict) concavity of $f(\exp (\cdot))$ implies that $\theta f(\theta)$ is (strictly) increasing.

Combining this fact with (10), it follows that

$$
\begin{aligned}
& \int_{\theta_{0}}^{\theta_{2}} \theta \hat{n}(\theta) f(\theta) d \theta-\int_{\theta_{0}}^{\theta_{2}} \theta \iota^{*}(\theta) n^{*}(\theta) f(\theta) d \theta \\
> & \theta_{0} f\left(\theta_{0}\right)\left(\int_{\theta_{0}}^{\theta_{2}} \hat{n}(\theta) d \theta-\int_{\theta_{0}}^{\theta_{2}} \iota(\theta) n^{*}(\theta) d \theta\right) \\
= & \theta_{0} f\left(\theta_{0}\right) \int_{\theta_{1}}^{\theta_{0}} n^{*}(\theta) d \theta \\
\geq & \int_{\theta_{1}}^{\theta_{0}} \iota(\theta) \theta n^{*}(\theta) f(\theta) d \theta
\end{aligned}
$$

which can be rearranged to

$$
\int_{\theta_{1}}^{\theta_{2}} \iota(\theta) \theta n^{*}(\theta) d F(\theta)<\int_{\theta_{0}}^{\theta_{2}} \theta \hat{n}(\theta) d F(\theta) .
$$

Proof of Corollary 2. The arguments in the proofs of the comparative statics results are all a simple application of the implicit function theorem.

In addition, define

$$
G\left(\theta_{1}, \Lambda, \theta_{0}\right)=\int_{\theta_{1}}^{F^{-1}(\Lambda)} c^{\prime-1}\left(\theta-\frac{\tau}{1+\tau} \frac{\Lambda-F(\theta)}{f(\theta)}\right) d \theta-S_{O}\left(F^{-1}(\Lambda)\right) .
$$

Proof. Equation (10) in Theorem 1 states that

$$
\frac{\partial G}{\partial b}=0
$$

Differentiating $G$ w.r.t. $b$ gives

$$
\frac{\partial G}{\partial b}=-\frac{\partial \theta_{1}}{\partial b} \cdot y\left(\theta_{\text {eff }}\right)-\int_{\theta_{1}}^{F^{-1}(\Lambda)} \frac{\partial \Lambda}{\partial b} \frac{\tau}{1+\tau} \frac{1}{f(\theta)} y^{\prime}\left(\theta-\frac{\tau}{1+\tau} \frac{\Lambda-F(\theta)}{f(\theta)}\right) d \theta+\frac{\partial \theta_{0}}{\partial b} y\left(\theta_{0}\right) .
$$

By the definition of $\theta_{0}$, an increase in $b$ leads to an increase in $\theta_{0}$. Hence, $\partial \theta_{0} / \partial b>0$.

In addition, we claim that

$$
\frac{\partial \theta_{1}}{\partial b}>(<) \Leftrightarrow \frac{\partial \Lambda}{\partial b} .
$$

To see this, note that $\theta_{\text {eff }}$ is unaffected by any change in $b$. By condition (8), it must then hold that

$$
\frac{\partial h_{\tau}\left(\theta_{1}, \Lambda\right)}{\partial \theta} \frac{\partial \theta_{1}}{\partial b}+\frac{\partial h_{\tau}\left(\theta_{1}, \Lambda\right)}{\partial \Lambda} \frac{\partial \Lambda}{\partial b}=0
$$


Since $\frac{\partial h_{\tau}\left(\theta_{1}, \Lambda\right)}{\partial \theta}>0$ by Lemma 8 and $\frac{\partial h_{\tau}\left(\theta_{1}, \Lambda\right)}{\partial \Lambda}<0$, claim (80) follows.

Now, plugging (79) and $\partial \theta_{0} / \partial b>0$ into (78) and applying (80), it follows that $\partial \theta_{1} / \partial b>0$ as well as $\partial \Lambda / \partial b>0$ which implies $\partial \theta_{2} / \partial b>0$.

\section{Proof of Corollary 3.}

Proof. First note from the definitions of $\theta_{0}$ and $\theta_{\text {eff }}$ that $\partial \theta_{0} / \partial R, \partial \theta_{\text {eff }} / \partial R>0$.

By condition (10), it must hold that

$$
\frac{\partial G}{\partial R}=-\frac{\partial \theta_{1}}{\partial R} \cdot y\left(\theta_{\text {eff }}\right)-\int_{\theta_{1}}^{F^{-1}(\Lambda)} \frac{\partial \Lambda}{\partial R} \frac{\tau}{1+\tau} \frac{1}{f(\theta)} y^{\prime}\left(\theta-\frac{\tau}{1+\tau} \frac{\Lambda-F(\theta)}{f(\theta)}\right) d \theta+\frac{\partial \theta_{0}}{\partial R} y\left(\theta_{0}\right)=0
$$

Now suppose that $\partial \theta_{1} / \partial R \leq 0$ which we will lead to a contradiction. By condition (8), we then have

$$
\frac{\partial h_{\tau}\left(\theta_{1}, \Lambda\right)}{\partial \theta} \frac{\partial \theta_{1}}{\partial R}+\frac{\partial h_{\tau}\left(\theta_{1}, \Lambda\right)}{\partial \Lambda} \frac{\partial \Lambda}{\partial R}-\frac{\partial \theta_{\text {eff }}}{\partial R}=0
$$

Following the argument as in the proof of Corollary 2, we obtain $\partial \Lambda / \partial R>0$. But then, $\partial G / \partial R>0$, a contradiction. Hence, $\partial \theta_{1} / \partial R>0$.

\section{Proof of Corollary 4 and Corollary 5.}

Proof. The results follow directly from the observation that $\lim _{\tau \rightarrow 0} \frac{\tau}{1+\tau}=0$ and $\lim _{\tau \rightarrow \infty} \frac{\tau}{1+\tau}=1$ respectively, and the fact that all endogenous variables $n, \iota$ and $\Lambda$ in Theorem 1 can be chosen from compact intervals. 


\section{B Further figures and tables}

\begin{tabular}{cccccccc}
\hline \multirow{2}{*}{$\tau$} & $u^{*}$ & $\begin{array}{c}\text { Average } \\
\text { hour } \\
\text { reduction }\end{array}$ & $\begin{array}{c}\text { Share in } \\
\text { STW }\end{array}$ & $\begin{array}{c}\text { Share of } \\
\text { transfers to } \\
\text { STW }\end{array}$ & $\begin{array}{c}\text { Average } \\
\text { hour } \\
\text { reduction } \\
\text { (adjusted) }\end{array}$ & $\begin{array}{c}\text { Share in } \\
\text { STW } \\
\text { (adjusted) }\end{array}$ & $\begin{array}{c}\text { Share of } \\
\text { transfers to } \\
\text { STW } \\
\text { (adjusted) }\end{array}$ \\
\hline 0.3 & 0.02 & 0.04 & $87.5 \%$ & $67.8 \%$ & 0.1 & $15.3 \%$ & $32.4 \%$ \\
& 0.04 & 0.03 & $77.7 \%$ & $67.2 \%$ & 0.09 & $13.4 \%$ & $32.6 \%$ \\
& 0.06 & 0.03 & $62.9 \%$ & $64.5 \%$ & 0.08 & $10.4 \%$ & $30.4 \%$ \\
0.5 & 0.02 & 0.05 & $54.9 \%$ & $51.4 \%$ & 0.1 & $15.2 \%$ & $35.9 \%$ \\
& 0.04 & 0.04 & $47.6 \%$ & $51.8 \%$ & 0.09 & $12.6 \%$ & $34.4 \%$ \\
& 0.06 & 0.04 & $38.8 \%$ & $50.3 \%$ & 0.08 & $9.5 \%$ & $30.7 \%$ \\
& 0.02 & 0.05 & $43.5 \%$ & $46.1 \%$ & 0.11 & $14.5 \%$ & $35.5 \%$ \\
& 0.04 & 0.05 & $38.5 \%$ & $47.1 \%$ & 0.1 & $12.3 \%$ & $34.4 \%$ \\
\hline
\end{tabular}

Table B.1: Moments under a counter-factual optimal short-time work policy. In columns 6-8, we adjust our definition of short-time work to matches which change hours by more than 0.05 units relative to hours in the outside option. Full-time work is normalized to 1. 


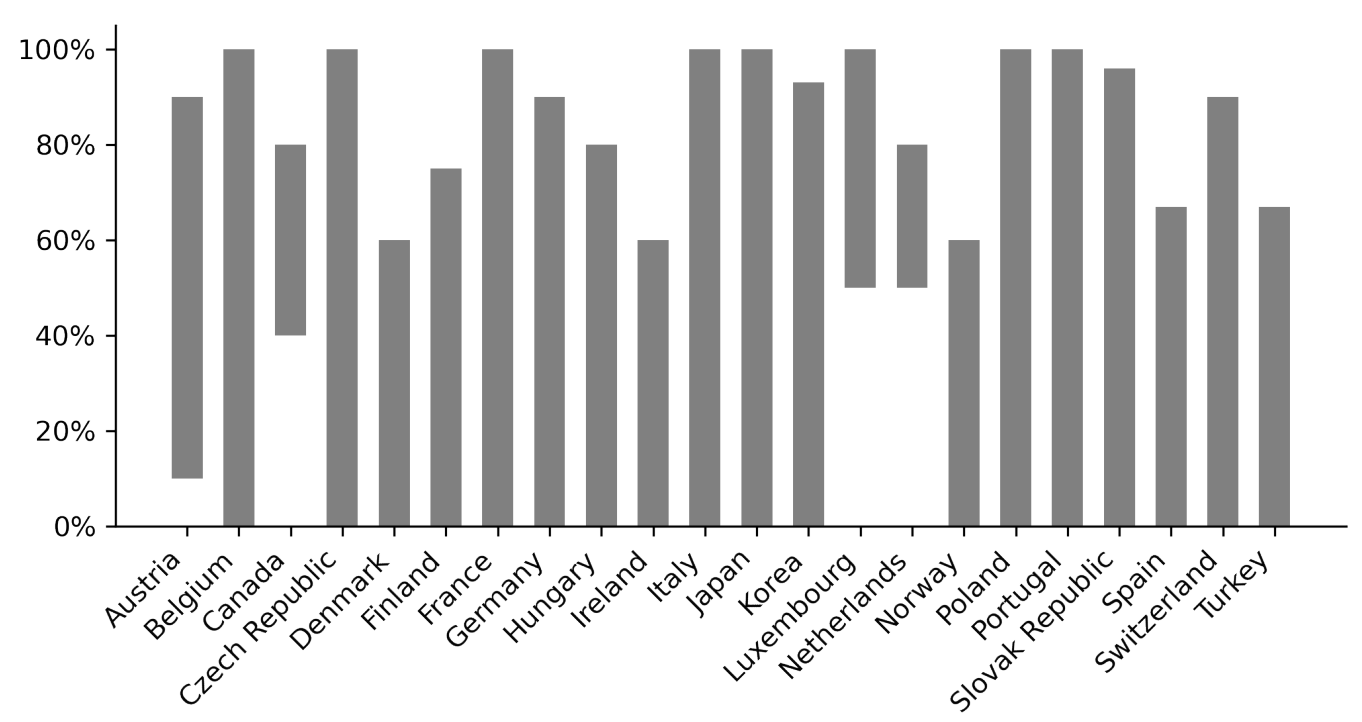

Figure B.1: Range of working hours relative to full-time work under STW policies in selected OECD countries in 2008-2009. Source: Hijzen and Venn (2011), based on reports to OECD delegates 


\section{Comparison with an employment subsidy without reduction of hours}

STW aims to sustain employment through a combination of transfers and a reduction of work hours. A common and related labor market policy are direct employment subsidies, which promise a transfer conditional on employment but without making amends for hours. In this section, we consider a special case of our model to understand under what conditions this simpler policy is preferable to STW. The example highlight the limits of hours as a screening tool and further illustrates the mechanism of our model.

In contrast to our standard model, we assume that the worker does not incur a convex disutility of labor. Instead, labor is provided at no cost up to an upper bound $\bar{n}=1$. Alternatively, one could assume that the disutility of labor becomes prohibitively large when hours are highter than the bound. We further simplify the model and assume that there is no benefits to reallocation, i.e. $R=0$. In this scenario, the surplus in the outside option for the match becomes

$$
\begin{aligned}
\tilde{S}_{O}(\theta) & =\max _{n \in[0, \bar{n}]} \mathbb{1}_{\{n>0\}}(J(\theta, n)-V+W(\theta, n)-U) \\
& =\max _{n \in[0,1]}
\end{aligned}
$$

with solution $\hat{\hat{n}}$

$$
\hat{\tilde{n}}(\theta)= \begin{cases}0, & \text { if } \theta \leq U \\ 1, & \text { if } \theta>U\end{cases}
$$

The planner instead offers surplus $\tilde{S}_{P}=\theta \times n+t(\theta)-U$ and solves

$$
\tilde{\mathcal{W}}=\int_{\theta}\left[\tilde{S}_{P}(\theta)-(1+\tau) t(\theta)\right] d F(\theta)
$$

subject to the usual incentive and participation constraints. Moreover, the planner also has to respect the hour constraint $n \in[0,1]$.

When the planner implements the outside option, the value of the problem is

$$
\hat{\mathcal{W}}=\int_{U}^{\bar{\theta}} \theta d F(\theta)-\tau \times U \times F(U),
$$

where a mass $1-F(U)$ of matches produce at maximal hours $\bar{n}=1$ and a mass of $F(U)$ of exiting matches cause excess costs to public funds of $\tau \times U$. Moreover, the outside option satisfies the incentive compatibility constraint since unemployment benefits $U$ provide no improvement for matches with $\theta \geq$ $U$.

As an improvement over this allocation, the planner could implement an incentive-compatible and individually rational employment subsidy by offering a policy

$$
(\tilde{n}(\theta), \tilde{t}(\theta))= \begin{cases}(0, U) & \text { if } \theta<U-\tilde{U} \\ (1, \tilde{U}) & \text { if } \theta \geq U-\tilde{U}\end{cases}
$$


with payoff for the planner of

$$
\tilde{\mathcal{W}}(\tilde{U})=\int_{U-\tilde{U}}^{\bar{\theta}} \theta d F(\theta)-\tau \times \tilde{U}-\tau \times(U-\tilde{U}) F(U-\tilde{U})
$$

All matches would participate in the program. Since the subsidy is flat in productivity $\theta$, all matches reveal their productivity truthfully. The planner solves for this trade-off by finding the optimal employment subsidy $\tilde{U}$ that maximizes

$$
\tilde{\mathcal{W}}=\max _{\tilde{U} \in[0, U]} \tilde{\mathcal{W}}(\tilde{U}) .
$$

Whether or not the employment protection program is an improvement depends on the relative weight of matches $1-F(U)$ above the exit-threshold who benefit from an employment subsidy without being at risk of termination, and matches below the exit-threshold $F(U)$ which would have been terminated without an employment subsidy. For an interior solution, the optimal subsidy $\tilde{U}$ is implicitly defined through the first order condition

$$
(1+\tau)\left(U-\tilde{U}^{\star}\right) f\left(U-\tilde{U}^{\star}\right)=\tau\left[1-F\left(U-\tilde{U}^{\star}\right)\right]
$$

where the assumption of monotone hazard rates guarantees a unique solution $\tilde{U}^{\star}$. Intuitively, the right hand side displays the marginal benefit the production plus the reduction in costs of unemployment of type $U-\tilde{U}$, whereas the term on the right represents the incentive costs. Since higher types can be neither excluded nor screened, a marginal extension of the subsidy $\tilde{U}$ has to be offered to all higher types.

This simple example highlights that the mass of matches which exit under autarky are an important statistic for the efficiency of short time work. In the presence of asymmetric information, an employment subsidy is only beneficial if many matches need support or, equivalently, few matches have to be compensated for truth-telling. Figure C.2 provides intuition for two cases. In panel C.2a on the left, a large mass of matches $1-F(U)$ do not exit without an employment subsidy and therefore have to be compensated for their private information. The incentive costs are too high and the social planner does not intervene. Formally, the optimal decision of the planner is to implement the outside option of the matches. In panel C.2b, a sufficiently large mass of matches $F(U)$ would exit absent any subsidy. The planner finds it optimal to subsidize matches up to a threshold productivity $U-\tilde{U}$ at the cost of compensating all producing matches with a flat subsidy $\tilde{U}$. 


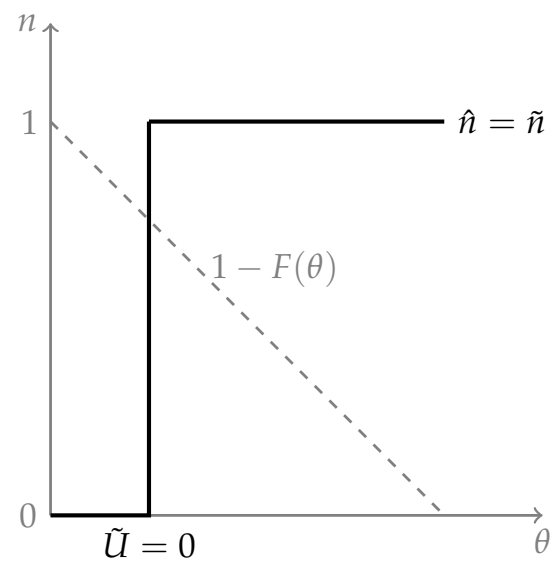

(a) Low unemployment. Most matches are allocated to the right of $U$.

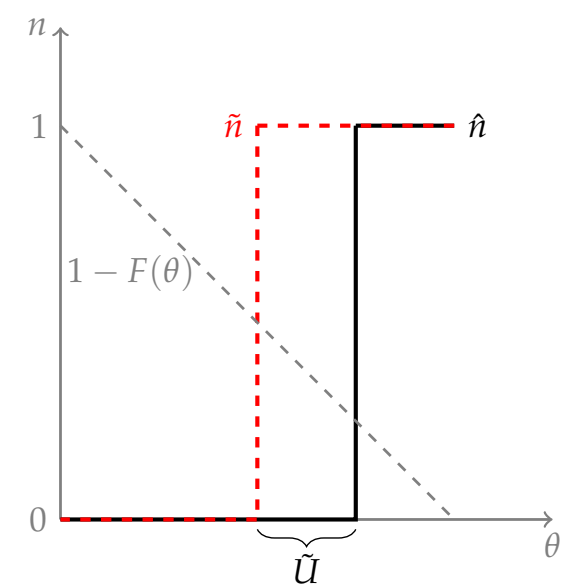

(b) High unemployment. Most matches are to the left of $U$.

Figure C.2: Example of a flat employment subsidy. In the left panel, the mass of firms that continues without a subsidy is large and incentive costs prevent the planner from saving matches. On the right, the mass of firms that would exit is large. The planner preserves matches up to cutoff $U-\tilde{U}$ at the costs of compensating high-productivity matches. 